\title{
ANALYSE QUASI-SURE ET L'ESTIMATION DU NOYAU DE LA CHALEUR POUR TEMPS PETIT
}

\author{
SHIZAN FANG
}

\begin{abstract}
The Ito functionals can be redefined out of a slim set by the natural way. Quasi-sure analysis is used to deal with the heat kernel asymptotic problems.
\end{abstract}

En 1982, R. S. Ellis et J. S. Rosen ont developpé une méthode de Laplace pour les intégrales gaussiennes de fonctions continues, différentiables au sens de calcul differentiel Banachique [E-R]. L'étude du noyau de la chaleur pour temps petit semble relever formellement de la même methodologie. Toutefois les théorèmes de $[E-R]$ ne peuvent pas s'appliquer, les fonctionnelles d'Ito définies sur l'espace de Wiener $X$ par les solutions d'équations différentielles stochastiques ne sont pas continues, a fortiori ne sont pas différentiables au sens du calcul différentiel Banachique.

Ce travail s'inscrit dans le cadre du calcul des variations stochastiques, cadre où les fonctionnelles d'Ito sont différentiables [ $\left.\mathrm{MA}_{1}\right]$, mais où les fonctionnelles d'Ito ne sont définies seulement presque partout. L'analyse quasi-sure permet de construire des redéfinitions de fonctionnelles d'Ito hors d'ensemble de capacité nulle pour une échelle infinie de capacité. Elle permet ainsi de travailler en codimension finie [ $\left.\mathbf{M A}_{2}\right]$ comme dans le calcul différentiel usuel. Par exemple, la loi d'une fonctonnelle d'Ito à valeurs dans $\mathbb{R}^{d}$ s'exprime par une intégrale sur la fibre relativement à la mesure d'aire $\mathscr{H}^{\infty-d}$.

Dans le cadre de l'analyse quasi sûre, un théorème des fonctions implicites en codimension finie peut être démontré $\left[\mathrm{MA}_{2}\right]$, la faiblesse de ce théorème est que l'on ne peut pas choisir arbitrairement le point où le théorème des fonctions implicites est appliqué. Pour pallier à cette difficulté, nous utiliserons la redéfinition canonique de Ren [RE]. Cette redéfinition a une valeur bien déterminée en tous les points de l'espace Cameron-Martin $H$, bien que $H$ soit de capacité nulle sur toute l'échelle des capacités. Alors il devient possible d'écrire un théorème des fonctions implicites en un point fixé $h_{0} \in H$.

Plus précisément, nous considérons l'équation différentielle stochastique de Stratonoviche:

$$
d \xi_{t}=\sum_{i=1}^{m} A_{i}\left(\xi_{t}\right) \circ d x^{i}(t), \quad \xi(0)=\xi_{0},
$$

où $A_{1}, \ldots, A_{m}$ sont $m$ champs de vecteurs sur $\mathbb{R}^{d}$, bornés à dérivées bornées.

Received by the editors July 9, 1990 and, in revised form, June 3, 1991.

1991 Mathematics Subject Classification. Primary 58G32, 60H07; Secondary 60H10. 
Le point $h_{0} \in H$ que nous choisissons sera la "géodésique minisante" pour l'action horizontale associée à l'opérateur de Hörmander $\frac{1}{2} \sum_{i=1}^{m} A_{i}^{2}$. Sous les hypothèses de Hörmander et de Bismut, un développement asymptotique de $p_{t}\left(\xi_{0}, \xi\right)$ (la loi du processus associé) sera obtenu pour temps petit lorsque $\xi_{0}$ et $\xi$ sont non conjugués. Pour ce faire, nous établirons un théorème des fonctions implicites sur un voisinage de $h_{0}$ uniforme en divers paramètres, notament $t$, utilisant en particulier les estimations de Moulinier [MO]. L'estimation à l'extérieur du domaine de définition de la fonction implicite sera obtenue par la théorie des grandes déviations $\left[\mathrm{AZ}_{1}\right.$ et $\left.\mathrm{ST}\right]$.

Diverses méthodes de Ben Arous [BE], Bismut [ $\left.\mathrm{BI}_{1}\right]$, Léandre [LE], et Watanabe $\left[\mathrm{WA}_{2}\right]$ existent déjà avant ce travail, pour approcher ce problème. La méthode présentée ici paraît la plus proche de la théorie classique en dimension finie des intégrales de Laplace et des méthodes géométriques de leur traitement. (Voir [BI ${ }_{1}$, Introduction et ER].)

Une version affaiblie des résultats de ce travail a été annoncé dans les Comptes Rendus [FA].

\section{RAPPELS ET NOTATIONS}

Soit $X$ l'espace de Wiener classique des fonctions continues de $[0,1]$ à valeurs dans $\mathbb{R}^{m}$, s'annulant à l'origine. On désigne par $H$ le sous espace Cameron-Martin de $X$ tel que $h \in H$ soit $d t$ p.p. dérivable et

$$
(h \mid h)_{H}=\int_{0}^{1}\|\dot{h}(t)\|^{2} d t<\infty,
$$

par $H_{0}$ le dual topologique de $X$ qui s'identifie à l'espace:

$$
H_{0}=\left\{h \in X \mid \ddot{h}_{i} \text { est une mesure sur }[0,1]\right\} .
$$

Soit $\mu$ la mesure de Wiener sur $X$.

On note par $\nabla$ (resp. $\mathscr{L}$ ) l'opérateur de gradient (resp. Ornstein-Uhlenbeck) sur $X$, l'espace de Sobolev $W_{2 r}^{p}$ est l'ensemble des fonctions telles que:

$$
\|\phi\|_{p, 2 r}^{p}=\int|\phi|^{p} d \mu+\sum_{i=1}^{2 r} \int\left\|\nabla^{i} \phi\right\|_{H_{\otimes^{i}}}^{p} d \mu<+\infty
$$

ou

$$
\|\phi\|_{W_{2 r}^{p}}^{p}=\int|\phi|^{p} d \mu+\int\left|\mathscr{L}^{r} \phi\right|^{p} d \mu<+\infty .
$$

On note $W_{\infty}\left(\mathbb{R}^{d}\right)$ l'intersection de tous les espaces de Sobolev sur $X$ à valeurs dans $\mathbb{R}^{d}$. Une fonction $\phi$ de $W_{\infty}\left(\mathbb{R}^{d}\right)$ est non dégénérée si:

$$
\operatorname{det} \phi(x)=\operatorname{det}\left(\left(\nabla \phi^{i} \mid \nabla \phi^{j}\right)_{H}\right)^{1 / 2}>0 \quad \mu \text {-p.p. et }[\operatorname{det} \phi]^{-1} \in W_{\infty}(\mathbb{R}) \text {. }
$$

Dans ce cas, la mesure image $\phi_{*} \mu$ est absolument continue par rapport à la mesure de Lebesgue $d \xi$ sur $\mathbb{R}^{d}$, on a $\phi_{*} \mu=k(\xi) d \xi$, avec $k \in \mathscr{C}^{\infty}\left(\mathbb{R}^{d}\right)$.

D'après [ $\left.\mathrm{MA}_{2}\right]$, la $(p, r)$-capacité d'un ensemble ouvert $O$ de $X$ est définie par:

$$
C_{p, r}(O)=\inf \left\{\|u\|_{W_{2 r}^{p}} ; u \geq 0, u \geq 1 \mu \text {-p.p. sur } O\right\}
$$

pour un ensemble quelconque $A \subset X$ :

$$
C_{p, r}(A)=\inf \left\{C_{p, r}(O) \mid O \text { ouvert } \supset A\right\} .
$$


L'ensemble $A$ est mince si $C_{p, r}(A)=0$ pour tour $p, r$. Une fonction $\phi$ de $W_{\infty}\left(\mathbb{R}^{d}\right)$ peut être redéfinie en dehors d'un ensemble mince, et cette redéfinition $\phi^{*}$ est unique à un ensemble mince près.

Soit $\phi \in W_{\infty}\left(\mathbb{R}^{d}\right)$ non dégénérée, d'après [A-M], il existe une mesure d'aire $\mathscr{H}^{\infty-d}$ sur $\phi^{*^{-1}}(\xi)$, ne chargeant aucun ensemble mince, telle que:

$$
k(\xi)=\int_{\phi^{-1}(\xi)} \frac{d \mathscr{H} \infty-d}{[\operatorname{det} \phi]^{*}(x)} .
$$

\section{REDÉFINITION DE REN}

Dans un travail récent de $[R E]$, Ren établit un théorème limite quasi-sûr de l'équation différentielle stochastique (0.1) par l'approximation de StroockVaradhan $[\mathrm{S}-\mathrm{V}]$ :

$$
d \xi_{t}^{\rho}(x)=\left[\sum_{i=1}^{m} A_{i}\left(\xi_{t}^{\rho}(x)\right) \dot{x}_{\rho}^{i}(t)\right] d t, \xi_{0}^{\rho}(x)=\xi_{0},
$$

où $\dot{x}_{\rho}^{i}(t)=\left(x\left(t_{\rho}^{+}\right)-x\left(t_{\rho}\right)\right) / \rho$ si $t \in\left[t_{\rho}, t_{\rho}^{+}\right]$, avec $t_{\rho}=[t / \rho]$ et $t_{\rho}^{+}=t_{\rho}+\rho$.

2.1. Notons $\Sigma^{0}=\left\{x \in X \mid \lim _{\rho \rightarrow 0} \xi_{t}^{\rho}(x)\right.$ existe uniformément en $\left.t \in[0,1]\right\}$, alors d'après [RE], $\Sigma^{0} \supset H$ et le complémentaire de $\Sigma^{0}$ dans $X$ est un ensemble mince. On notera $\varphi_{t}(x)$ la redéfinition ainsi obtenue.

Soit $H_{2} \subset H_{0}$ un sous espace de dimension finie, $d=\operatorname{dim} H_{2}$, soit $e_{1}, \ldots, e_{d}$ une base orthonormée de $\mathrm{H}_{2}$, c'est-à-dire:

$$
\int_{0}^{1} \sum_{k=1}^{m} \dot{e}_{i}^{k}(t) \dot{e}_{j}^{k}(t) d t=\delta_{i j} \quad \text { (le symbole de Kronenker). }
$$

On suppose de plus que $t \rightarrow e_{i}(t) \quad(i=1, \ldots, d)$ sont des fonctions $\mathscr{C}^{\infty}$. Une fois $e_{1}, \ldots, e_{d}$ choisies, $H_{2}$ est isométrique à $\mathbb{R}^{d}$ munie de la norme euclidienne.

Soit $0<\varepsilon \leq 1, h \in \mathrm{H}_{2}$, on considère l'équation différentielle stochastique:

$$
\begin{aligned}
d \xi_{\varepsilon, h}(t) & =\sum_{i=1}^{m} \varepsilon A_{i}\left(\xi_{\varepsilon, h}\right) \circ d x^{i}(t)+\sum_{i=1}^{m} A_{i}\left(\xi_{\varepsilon, h}\right) \dot{h}^{i}(t) d t, \\
\xi_{\varepsilon, h}(0) & =\xi_{0}
\end{aligned}
$$

et sa régularisation de Stroock-Varadhan:

$$
\begin{aligned}
d \xi_{\varepsilon, h}^{\rho}(t) & =\sum_{i=1}^{m}\left[\varepsilon A_{i}\left(\xi_{\varepsilon, h}^{\rho}\right) \dot{x}_{\rho}^{i}(t)+A_{i}\left(\xi_{\varepsilon, h}^{\rho}\right) \dot{h}_{\rho}^{i}(t)\right] d t, \\
\xi_{\varepsilon, h}^{\rho}(0) & =\xi_{0} .
\end{aligned}
$$

Théorème 2.1. Soit $R>0$, il existe un ensemble $\Sigma_{R} \subset X$ dont le complémentaire est mince dans $X$ tel que pour tout $x \in \Sigma_{R}, \xi_{\varepsilon, h}^{\rho}(t, x)(\rho \rightarrow 0)$ converge uniformément vers $\xi_{\varepsilon, h}(t, x)$ en $(t, \varepsilon) \in[0,1] \times[0,1]$, et en $h \in \overline{B_{H_{2}}(0, R)}=$ $\left\{h \in H_{2} \mid\|h\|_{H} \leq R\right\}$.

Le démonstration de ce résultat repose sur la version de Ren du théorème de Kolmogoroff pour la convergence quasi-sûre [RE, p. 192] et des lemmes suivants. 


\section{Lemme 2.2.}

(2.5)

$$
E\left(\left|\xi_{\varepsilon, h}^{\rho}(t)-\xi_{\varepsilon^{\prime}, h}^{\rho}(t)\right|^{2 p}\right) \leq C\left|\varepsilon-\varepsilon^{\prime}\right|^{2 p} \quad \text { si } h \in \overline{B_{H_{2}}(0, R)} .
$$

Démonstration. On a:

$$
\begin{aligned}
\xi_{\varepsilon, h}^{\rho}(t, x)-\xi_{\varepsilon^{\prime}, h}^{\rho}(t, x)= & \left.\sum_{i=1}^{m}\left[\int_{0}^{t} \varepsilon A_{i}\left(\xi_{\varepsilon, h}^{\rho}(s)\right)-\varepsilon^{\prime} A_{i}\left(\xi_{\varepsilon^{\prime}, h}^{\rho}(s)\right)\right] \dot{x}_{\rho}^{i}(s) d s\right] \\
& +\sum_{i=1}^{m} \int_{0}^{t}\left[A_{i}\left(\xi_{\varepsilon, h}^{\rho}\right)-A_{i}\left(\xi_{\varepsilon^{\prime}, h}^{\rho}\right)\right] \dot{h}_{\rho}^{i}(s) d s \\
= & I_{1}+I_{2} .
\end{aligned}
$$

On a:

$$
\begin{aligned}
\left|I_{2}\right| & \leq \sum_{i=1}^{m} \int_{0}^{t}\left|\left[A_{i}\left(\xi_{\varepsilon, h}^{\rho}\right)-A_{i}\left(\xi_{\varepsilon^{\prime}, h}^{\rho}\right)\right]\right|\left|\dot{h}_{\rho}^{i}(s)\right| d s \\
& \leq \sum_{i=1}^{m} C \int_{0}^{t}\left\|\xi_{\varepsilon, h}^{\rho}(s)-\xi_{\varepsilon^{\prime}, h}^{\rho}(s)\right\| \cdot\left|\dot{h}_{\rho}^{i}(s)\right| d s .
\end{aligned}
$$

Rappelons que $\dot{h}_{\rho}^{1}(s)=\left(h^{i}(s)-h^{i}\left(s_{\rho}\right)\right) / \rho$, et $\left|s-s_{\rho}\right| \leq \rho$. En posant:

$$
N(h)=\sup _{0 \leq t \leq 1} \sum_{i=1}^{m}\left|\dot{h}^{i}(t)\right|
$$

on a:

$$
\sum_{i=1}^{m}\left|\dot{h}_{\rho}^{i}(s)\right| \leq \sum_{i=1}^{m} \frac{1}{\rho} \int_{s_{\rho}}^{s}\left|\dot{h}^{i}(t)\right| d t \leq N(h) .
$$

Il est clair que $N(h)$ est une norme sur $H_{2}$, elle est donc équivalente à $\|h\|_{H}$, autrement dit, il existe $C>0$ telle que:

$$
(1 / C)\|h\|_{H} \leq N(h) \leq C\|h\|_{H}, \quad h \in H_{2} .
$$

On a donc:

$$
\left|I_{2}\right| \leq C\|h\|_{H} \int_{0}^{t}\left\|\xi_{\varepsilon, h}^{\rho}(s)-\xi_{\varepsilon^{\prime}, h}^{\rho}(s)\right\| d s
$$

d'où:

$$
E\left(\left|I_{2}\right|^{2 p}\right) \leq(C R)^{2 p} \int_{0}^{t} E\left(\left\|\xi_{\varepsilon, h}^{\rho}(s)-\xi_{\varepsilon^{\prime}, h}^{\rho}(s)\right\|^{2 p}\right) d s .
$$

L'estimation de $I_{1}$ est plus délicate, on va suivre des idées de Moulinier [MO]:

$$
\begin{aligned}
I_{1}= & \sum_{i=1}^{m} \int_{0}^{t} \varepsilon\left[A_{i}\left(\xi_{\varepsilon, h}^{\rho}\right)-A_{i}\left(\xi_{\varepsilon^{\prime}, h}^{\rho}\right)\right] \dot{x}_{\rho}^{i}(s) d s \\
& +\sum_{i=1}^{m}\left(\varepsilon-\varepsilon^{\prime}\right) \int_{0}^{t} A_{i}\left(\xi_{\varepsilon^{\prime}, h}^{\rho}\right) \dot{x}_{\rho}^{i}(s) d s \\
= & I_{11}+I_{12} .
\end{aligned}
$$


On a:

$$
\begin{aligned}
I_{12}= & \left(\varepsilon-\varepsilon^{\prime}\right) \sum_{i=1}^{m}\left[\int_{0}^{t} A_{i}\left(\xi_{\varepsilon^{\prime}, h}^{\rho}\left(s_{\rho}\right)\right) \dot{x}_{i}^{i}(s) d s\right. \\
& \left.+\int_{0}^{t}\left[A_{i}\left(\xi_{\varepsilon^{\prime}, h}^{\rho}(s)\right)-A_{i}\left(\xi_{\varepsilon^{\prime}, h}^{\rho}\left(s_{\rho}\right)\right)\right] \dot{x}_{\rho}^{i}(s) d s\right] \\
= & \left(\varepsilon-\varepsilon^{\prime}\right)\left[I_{121}+I_{122}\right] .
\end{aligned}
$$

Le terme $I_{121}=\sum_{i=1}^{m} \int_{0}^{t} A_{i}\left(\xi_{\varepsilon^{\prime}, h}^{\rho}\right) \dot{x}_{\rho}^{i}(s) d s$ est une martingale, d'après l'inégalité de Burkholder-Davis:

$$
E\left(\left|I_{121}\right|^{2 p}\right) \leq C \int_{0}^{t} E\left(\left\|A_{i}\left(\xi_{\varepsilon^{\prime}, h}^{\rho}\left(s_{\rho}\right)\right)\right\|^{2}\right)^{p} d s \leq C_{1} .
$$

On a:

$$
\begin{gathered}
\left|I_{122}\right| \leq C \int_{0}^{t}\left\|\xi_{\varepsilon^{\prime}, h}^{\rho}(s)-\xi_{\varepsilon^{\prime}, h}^{\rho}\left(s_{\rho}\right)\right\|\left|\dot{x}_{\rho}^{i}(s)\right| d s \\
E\left(\left|\dot{x}_{\rho}^{i}\right|^{2 p}\right) \leq C \rho^{-p} \\
\xi_{\varepsilon^{\prime}, h}^{\rho}(s)-\xi_{\varepsilon^{\prime}, h}^{\rho}\left(s_{\rho}\right) \\
=\sum_{i=1}^{m}\left[\dot{x}_{\rho}^{i}(s) \varepsilon^{\prime} \int_{s_{\rho}}^{s} A_{i}\left(\xi_{\varepsilon^{\prime}, h}^{\rho}(u)\right) d u+\dot{h}_{\rho}^{i}(s) \int_{s_{\rho}}^{s} A_{i}\left(\xi_{\varepsilon^{\prime}, h}^{\rho}(u)\right) d u\right], \\
\sup _{0 \leq t \leq 1}\left|h_{\rho}^{i}\right| \leq \int_{0}^{1}\left|\dot{h}_{\rho}^{i}(t)\right| d t \leq N(h) \leq C\|h\|_{H} .
\end{gathered}
$$

Compte tenu de (2.7)-(2.10), on a $E\left(\left|I_{122}\right|^{2 p}\right) \leq C$. En le combinant avec (2.6), on obtient:

$$
E\left(\left|I_{12}\right|^{2 p}\right) \leq C\left|\varepsilon-\varepsilon^{\prime}\right|^{2 p}
$$

Quant au terme $I_{11}$, on suit les méthodes de [MO] en faisant quelques modifications mineures et on obtient:

$$
E\left(\left|I_{11}\right|^{2 p}\right) \leq C\left(\left|\varepsilon-\varepsilon^{\prime}\right|^{2 p}+\int_{0}^{t} E\left(\left\|\xi_{\varepsilon, h}^{\rho}\left(s_{\rho}\right)-\xi_{\varepsilon^{\prime}, h}^{\rho}\left(s_{\rho}\right)\right\|^{2 p}\right) d s\right) .
$$

Finalement:

$$
\begin{aligned}
E\left(\left\|\xi_{\varepsilon, h}^{\rho}(t)-\xi_{\varepsilon^{\prime}, h}^{\rho}(t)\right\|^{2 p}\right) \leq C\left\{\left|\varepsilon-\varepsilon^{\prime}\right|^{2 p}\right. & +\int_{0}^{t} E\left(\left\|\xi_{\varepsilon, h}^{\rho}(s)-\xi_{\varepsilon^{\prime}, h}^{\rho}(s)\right\|^{2 p}\right) d s \\
& \left.+\int_{0}^{t} E\left(\left\|\xi_{\varepsilon, h}^{\rho}\left(s_{\rho}\right)-\xi_{\varepsilon^{\prime}, h}^{\rho}\left(s_{\rho}\right)\right\|^{2 p}\right) d s\right\} .
\end{aligned}
$$

On en déduit que

$$
E\left(\left\|\xi_{\varepsilon, h}^{\rho}(t)-\xi_{\varepsilon^{\prime}, h}^{\rho}(t)\right\|^{2 p}\right) \leq C\left|\varepsilon-\varepsilon^{\prime}\right|^{2 p}
$$

Lemme 2.3. On $a$ :

$$
E\left(\left\|\xi_{\varepsilon, h}^{\rho}(t)-\xi_{\varepsilon, h}^{\rho},(t)\right\|^{2 p}\right) \leq C\left\|h-h^{\prime}\right\|_{H}^{2 p} \quad \text { si } h, h^{\prime} \in \overline{B_{H_{2}}(0, R)} .
$$

Démonstration. Méthode analogue.

En utilisant les Lemmes 2.2, 2.3 et [MO], on obtient: 
Lemme 2.4. Pour tout $p>10, R>0$, il existe $C>0$, telle que:

$$
\begin{aligned}
& E\left(\left\|\xi_{\varepsilon, h}^{\rho}(t)-\xi_{\varepsilon^{\prime}, h^{\prime}}^{\rho^{\prime}}\left(t^{\prime}\right)\right\|^{2 p}\right) \\
& \quad \leq C\left\{\left|t-t^{\prime}\right|^{p}+\left|\rho-\rho^{\prime}\right|^{p / 10}+\left\|h-h^{\prime}\right\|_{H}^{2 p}+\left|\varepsilon-\varepsilon^{\prime}\right|^{2 p}\right\}
\end{aligned}
$$

pour tout $\rho, \rho^{\prime}, \varepsilon, \varepsilon^{\prime} \in[0,1], h, h^{\prime} \in \overline{B_{H_{2}}(0, R)}$.

Démonstration du Théorème 2.1. D'après [RE], on a:

$$
\sup _{0<\rho<1} E\left[\sup _{(t, \varepsilon, h) \in K}\left\{\left|\xi_{\varepsilon, h}^{\rho}(t)\right|^{p}+\left|\mathscr{L}^{r} \xi_{\varepsilon, h}^{\rho}(t)\right|^{p}\right\}\right]<+\infty
$$

où $K=[0,1] \times[0,1] \times \overline{B_{H_{2}}(0, R)}$.

Pour $n>2 r, f \in W_{\infty}, p \geq 1$, il existe une constante $C(n, p, r)$ telle que:

$$
\begin{aligned}
\left\|f^{n}\right\|_{p, 2 r} \leq & C(n, p, r) \cdot \sum_{\alpha=1}^{2 r} \sum_{v^{\alpha}}\left[E\left(|f|^{(n-\alpha)(2 r+1) p}\right)\right]^{1 /(2 r+1) p} \\
& \times E\left(\|\nabla f\|_{H}^{v_{1}^{\alpha}(2 r+1) p}\right)^{1 /(2 r+1) p} \cdots E\left(\left\|\nabla^{2 r} f\right\|_{H_{\circledast 2}}^{v_{2 r}^{\alpha}(2 r+1) p}\right)^{1 /(2 r+1) p}
\end{aligned}
$$

où $v^{\alpha} \in \mathbb{N}^{2 r}$ vérifient les relations suivantes:

$$
v_{1}^{\alpha}+\cdots+v_{2 r}^{\alpha}=\alpha, \quad v_{1}^{\alpha}+2 v_{2}^{\alpha}+\cdots+2 r v_{2 r}^{\alpha}=2 r .
$$

Donc, d'après le Lemme 2.4, pour $n$ pair assez grand:

$$
\begin{aligned}
& \|\| \xi_{\varepsilon, h}^{\rho}(t)-\xi_{\varepsilon^{\prime}, h^{\prime}}^{\rho^{\prime}}\left(t^{\prime}\right)\left\|^{n}\right\|_{p, 2 r} \\
& \quad \leq C\left(\left|\rho-\rho^{\prime}\right|^{3+d+\alpha}+\left|t-t^{\prime}\right|^{3+d+\alpha}+\left|\varepsilon-\varepsilon^{\prime}\right|^{3+d+\alpha}+\left\|h-h^{\prime}\right\|_{H}^{3+d+\alpha}\right)
\end{aligned}
$$

avec $\alpha>0$.

Remarque. On a le même résultat pour les dérivées d'ordre supérieur en $\xi_{0}$.

Corollaire 2.5. Il existe un ensemble $\Sigma_{R}$ dont le complémentaire dans $X$ est un ensemble mince, $\Sigma_{R} \supset H$ tel que

(1) $\varepsilon \Sigma_{R}+\overline{B_{H_{2}}(0, R)} \subset \Sigma^{0}$,

(2) $\xi_{t}(\varepsilon x+h)=\xi_{\varepsilon, h}(t, x)$ si $x \in \Sigma_{R},(t, \varepsilon) \in[0,1]^{2}, h \in \overline{B_{H_{2}}(0, R)}$,

même résultat pour des dérivées d'ordre supérieurs en $\xi_{0}$.

Démonstration. Soit $\rho>0, \xi_{\varepsilon, h}^{\rho}(t, x)$ et $\xi_{t}^{\rho}(\varepsilon x+h)$ vérifient la même équation différentielle ordinaire:

$$
d u_{t}=\sum_{i=1}^{m}\left[\sigma A_{i}\left(u_{t}\right) \dot{x}_{\rho}^{i}(t)+A_{i}\left(u_{t}\right) \dot{h}_{\rho}^{i}(t)\right] d t, \quad u_{0}=\xi_{0} .
$$

Donc, $\xi_{\varepsilon, h}^{\rho}(t, x)=\xi_{t}^{\rho}(\varepsilon x+h)$. D'après le Théorème 2.1, il existe $\Sigma_{R}$ dont le complémentaire dans $X$ est mince, $\Sigma_{R} \supset H$ tel que, pour tout $x \in \Sigma_{R}$, $\xi_{\varepsilon, h}^{\rho}(t, x)$ converge uniformément en $t \in[0,1]$, pour tout $\varepsilon \in[0,1], h \in$ $\overline{B_{H_{2}}(0, R)}$, autrement dit, $\lim _{\rho \rightarrow 0} \xi_{t}^{\rho}(\varepsilon x+h)=\xi_{t}(\varepsilon x+h)$ uniformément en $t \in[0,1]$, donc $\varepsilon x+h \in \Sigma^{0}$. On obtient en même temps (1) et (2).

Notons désormais: $\xi_{t}^{n}(x)=\xi_{t}^{2^{-n}}(x), \phi_{n}(x)=\xi_{1}^{n}(x), \varphi(x)=\varphi_{1}(x)$,

$$
\varphi_{\varepsilon}(x)=\xi_{\varepsilon, 0}(1, x) \quad \text { et } \quad Z_{t}^{n}(x)=\partial \xi_{t}^{n}(x) / \partial \xi_{0}, \quad Z_{t}(x)=\partial \varphi_{t}(x) / \partial \xi_{0} .
$$


Proposition 2.6. Pour tout $x \in \Sigma_{R}, \nabla \phi_{n}(\varepsilon x+h)$ converge uniformément vers $\nabla \varphi(\varepsilon x+h)$ en $\varepsilon \in[0,1], h \in \widehat{B_{H_{2}}(0, R)}$.

Démonstration. Soient $m, n \geq 1$, posons $l_{m, n}=\nabla \phi_{m}-\nabla \phi_{n}$, on a:

$$
\begin{aligned}
{\left[\dot{l}_{m, n}(x)\right]^{\alpha}(t)=} & \int_{0}^{t} Z_{1}^{m}(x)\left(Z_{s}^{m}\right)^{-1}(x) A_{\alpha}\left(\xi_{s}^{m}(x)\right) d s \\
& -\int_{0}^{t} Z_{1}^{n}(x)\left(Z_{s}^{n}\right)^{-1}(x) A_{\alpha}\left(\xi_{s}^{n}(x)\right) d s .
\end{aligned}
$$

D'après le Théorème 2.1 , soit $\eta>0$, il existe $n_{0} \geq 1$ tel que pour tout $m, n \geq$ $n_{0}$, ait:

$$
\begin{aligned}
& \| Z_{1}^{m}(\varepsilon x+h)\left(Z_{s}^{m}\right)^{-1}(\varepsilon x+h) A_{\alpha}\left(\xi_{s}^{m}(\varepsilon x+h)\right) \\
& \quad-Z_{1}^{n}(\varepsilon x+h)\left(Z_{s}^{n}\right)^{-1}(\varepsilon x+h) A_{\alpha}\left(\xi_{s}^{n}(\varepsilon x+h)\right) \| \leq \eta
\end{aligned}
$$

pour tout $s \in[0,1], \varepsilon \in[0,1], h \in \overline{B_{H_{2}}(0, R)}$. Donc on a, en vertu de

$$
\left\|l_{m, n}(\varepsilon x+h)\right\|_{H \otimes \mathbb{R}^{d}}^{2} \leq \eta
$$

on en déduit la Proposition 2.5.

Dans ce qui suit, on va utiliser la méthode d'Egoroff pour passer de la convergence simple à la convergence uniforme.

Proposition 2.7. Soit $G$ un espace hilbertien séparable, $f_{n}^{\varepsilon, h}$ une suite de fonctions continues de $X$ dans $G$, dépendant de deux paramètres $(\varepsilon, h) \in[0,1] \times$ $\overline{B_{H_{2}}(0, R)}$. Supposons:

(1) $f_{n}^{\ell, h} \in W_{\infty}(X, G)$,

(2) pour tout $x \in \Sigma_{R}, f_{n}^{\varepsilon, h}(x)$ converge uniformément en $\varepsilon \in[0,1], h \in$ $\overline{B_{H_{2}}(0, R)}$.

Alors, pour tout $\delta>0$, il existe un ouvert $O \subset X$ tel que

(3) $C_{p, r}(O) \leq \delta$,

(4) $f_{n}^{\ell, h}(x)$ converge uniformément en $\varepsilon \in[0,1], h \in \overline{B_{H_{2}}(0, R)}, x \in O^{c}$.

Démonstration. Soit $\delta>0, l, k \in \mathbb{N}^{*}$, posons

$$
\begin{gathered}
S(l, k)=\bigcap_{m, n \geq l}\left\{x \in X \mid\left\|f_{n}^{\varepsilon, h}(x)-f_{m}^{\varepsilon, h}(x)\right\| \leq 1 / k, \forall \varepsilon \in[0,1], h \in \overline{B_{H_{2}}(0, R)}\right\}, \\
S_{0}=\left\{x \in X \mid f_{n}^{\varepsilon, h}(x) \text { ne converge pas uniformément en } \varepsilon \in[0,1],\right. \\
\left.h \in \overline{B_{H_{2}}(0, R)}\right\} .
\end{gathered}
$$

Par l'hypothèse (2), $\forall k \in \mathbb{N}^{*}$,

$$
S_{0}^{c} \subset \bigcup_{l} S(l, k), \quad S_{0}^{c}=\Sigma_{R}
$$

on a donc:

$$
C_{p, r}\left(\bigcap_{l} S^{c}(l, k)\right) \leq C_{p, r}\left(S_{0}\right)=C_{p, r}\left(\Sigma_{R}^{c}\right)=0
$$

$S^{c}(l, k)$ étant un ouvert de $X$, on obtient

$$
C_{p, r}\left(S^{c}\left(l_{k}, k\right)\right) \leq \delta / 2^{k} \text { pour un certain } l_{k} \text {. }
$$


Posons $O=\bigcup_{k \geq 1} S^{c}\left(l_{k}, k\right)$. On a:

$$
C_{p, r}(O) \leq \sum_{k \geq 1} C_{p, r}\left(S^{c}\left(l_{k}, k\right)\right) \leq \sum_{k \geq 1} 2^{-k} \delta=\delta .
$$

D'autre part, on voit facilement que $f_{n}^{\varepsilon, h}$ converge uniformément en $\varepsilon \in$ $[0,1], h \in \overline{B_{H_{2}}(0, R)}, x \in O^{c}$; la démonstration de la proposition est achevée.

Remarque. (1) $\xi_{t}(h)$ vérifie l'équation différentielle ordinaire suivante:

$$
d \xi_{t}(h)=\sum_{i=1}^{m} A_{i}\left(\xi_{t}(h)\right) \dot{h}^{i}(t) d t, \quad \xi_{0}(h)=\xi_{0},
$$

et $\xi_{t}^{n}(h), \nabla \xi_{t}^{n}(h), \ldots, \nabla^{r} \xi_{t}^{n}(h)$ converge uniformément sur tout borné de $H$. 0 .

(2) Notant $V(0, \rho)=\left\{x \in X \mid\|x\|_{\infty}<\rho\right\}$, on a $\lim _{\rho \rightarrow \infty} C_{p, r}\left(V^{c}(0, \rho)\right)=$

En résumant les résultats précédents, on obtient:

Théorème 2.8. Pour tout $R>0$, posons $B_{H}(0, R)=\left\{h \in H \mid\|h\|_{H}>R\right\}$, alors il existe une suite d'ouverts $O_{n, R, R^{\prime}} \subset X$ décroissants en $n, R$, croissant en $R^{\prime}$, et une suite de nombres $\rho_{n}>0$ tels que

(1) $B_{H}(0, R) \subset O_{n, R, R^{\prime}}^{c} \subset V\left(0, \rho_{n}\right)$;

(2) $\lim _{n \rightarrow \infty} C_{n, n}\left(O_{n, R, R^{\prime}}\right)=0$, pour tout $R, R^{\prime}$ fixés;

(3) $\phi_{m}(\varepsilon x+h), \ldots, \nabla^{r} \phi_{m}(\varepsilon x+h)$ converge uniformément lorsque $m \rightarrow \infty$ sur $[0,1] \times \overline{B_{H_{2}}\left(0, R^{\prime}\right)} \times O_{n, R, R^{\prime}}^{c}$.

Corollaire 2.9. Posons $G_{n, R, R^{\prime}}^{c}=\bigcup_{\varepsilon \in[0,1]} \varepsilon O_{n, R, R^{\prime}}^{c}$ et $Q_{n, R, R^{\prime}}^{c}=G_{n, R, R^{\prime}}^{c}+$ $\overline{B_{H_{2}}\left(0, R^{\prime}\right)}$; alors:

(1) $Q_{n, R, R^{\prime}}^{c}, G_{n, R, R^{\prime}}^{c}$ sont des fermés de $X$;

(2) $G_{n, R, R^{\prime}}^{c}$ est étoilé à $O$;

(3) $\phi_{m}, \ldots, \nabla^{r} \phi_{m}$ converge uniformément sur $Q_{n, R, R^{\prime}}^{c}$.

Démonstration. (1) Soit $x_{k} \in G_{n, R, R^{\prime}}^{c}$ tel que $\lim _{k \rightarrow \infty} x_{k}=x$, il existe $\varepsilon_{k} \in$ $[0,1], x_{k}^{\prime} \in O_{n, R, R^{\prime}}^{c}$ tels que $x_{k}=\varepsilon_{k} x_{k}^{\prime}$. Il existe une sous suite $k_{p}$ telle que $\lim _{p \rightarrow \infty} \varepsilon_{k_{p}}=\varepsilon$ avec $\varepsilon \in[0,1]$, si $\varepsilon=0$, d'après (1) du théorème précédent, $\lim _{p \rightarrow \infty} \varepsilon_{k_{p}} x_{k_{p}}^{\prime}=0$, on a $\lim _{p \rightarrow \infty} x_{k_{p}}=0$, donc $x=0 \in O_{n, R, R^{\prime}}^{c}$. Si $\varepsilon>0$, $\lim _{p \rightarrow \infty} x_{k_{p}}^{\prime}=\lim _{p \rightarrow \infty} x_{k_{p}} / \varepsilon_{k_{p}} \in O_{n, R, R^{\prime}}^{c}$, donc $x \in \varepsilon O_{n, R, R^{\prime}}^{c} \subset G_{n, R ; R^{\prime}}^{c}$ par conséquent $G_{n, R ; R^{\prime}}^{c}$ est fermé dans $X . \overline{B_{H_{2}}\left(0, R^{\prime}\right)}$ étant compact, il en résulte que $Q_{n, R, R^{\prime}}^{c}$ est fermé. (2) et (3) se voit par la construction.

\section{ESTIMATION DU NOYAU DE LA CHALEUR}

On suppose désormais que les champs de vecteurs $A_{1}, \ldots, A_{m}$ satisfont aux conditions fortes de Hörmander: $\mathbb{R}^{d}$.

(H.1) $A_{1}, \ldots, A_{m}$ et leurs crochets de Lie de tout ordre en $\xi_{0}$ engendrent

Dans ce cas, la solution $\xi_{t}$ de l'équation (0.1) est non dégénérée, on note $p_{t}\left(\xi_{0}, \xi\right)$ la densité de $\left(\xi_{t}\right)_{*} \mu$ par rapport à la mesure de Lebesgue $d \xi$ sur $\mathbb{R}^{d}$ et $p\left(\varepsilon, \xi_{0}, \xi\right)$ la densité de $\left(\varphi_{\varepsilon}\right)_{*} \mu$ relative à $d \xi$, on a la relation: $p_{\varepsilon^{2}}\left(\xi_{0}, \xi\right)=$ $p\left(\varepsilon, \xi_{0}, \xi\right)$. 
D'après $[\mathrm{A}-\mathrm{M}]$ :

$$
p\left(\varepsilon, \xi_{0}, \xi\right)=\int_{\varphi_{\varepsilon}^{-1}(\xi)} \frac{d \mathscr{H}^{\infty-d}}{\operatorname{det}\left[\varphi_{\varepsilon}\right]} .
$$

Soient $\xi_{0} \neq \xi$, notons:

$$
K_{\xi_{0}}^{\xi}=\{h \in H \mid \varphi(h)=\xi\} \quad \text { et } \quad d\left(\xi_{0}, \xi\right)=\inf \left\{\|h\|_{H} \mid h \in K_{\xi_{0}}^{\xi}\right\} .
$$

On supposera que la condition de Bismut est satisfaite:

(H.2) Il existe un seul $h_{0} \in K_{\xi_{0}}^{\xi}$ tel que $\left\|h_{0}\right\|_{H}=d\left(\xi_{0}, \xi\right)$ et $C_{h_{0}}$ (la matrice de covariance de Malliavin déterministe) inversible où:

$$
\left\langle C_{h} \eta, \eta\right\rangle=\sum_{i=1}^{m} \int_{0}^{1}\left\langle Z_{1}(h) Z_{t}^{-1}(h) A_{i}\left(\xi_{t}(h)\right), \eta\right\rangle^{2} d t, \quad \eta \in \mathbb{R}^{d} .
$$

Décomposition de l'espace de Wiener. Sous l'hypothèse (H.2), $t \rightarrow h_{0}(t)$ est une fonction $\mathscr{C}^{\infty}$ de $t$. Posons:

$$
\dot{e}_{i}^{\alpha}(t)=\left[Z_{1}\left(h_{0}\right) Z_{t}^{-1}\left(h_{0}\right) A_{\alpha}\left(\xi_{t}\left(h_{0}\right)\right)\right]^{i}, \quad i=1, \ldots, d .
$$

Alors les fonctions $t \rightarrow \dot{e}_{i}^{\alpha}(t) \quad(i=1, \ldots, d)$ sont des fonctions de $\mathscr{C}^{\infty}$ de $t$. Notons:

$$
H_{2}=\operatorname{vect}\left\{e_{1}, \ldots, e_{d}\right\} \quad \text { l'espace vectoriel engendré par } e_{1}, \ldots, e_{d} .
$$

$H_{2}$ est alors un sous espace de $H_{0}$ de dimension $d$, car $\left(\left(e_{i} \mid e_{j}\right)_{H}\right)=C_{h_{0}}$ est inversible. D'après $\left[\mathrm{BI}_{1}\right]$, il existe $q \in \mathbb{R}^{d}$ tel que:

$$
\dot{h}_{0}^{\alpha}(t)=\sum_{i=1}^{d} q_{i} e_{i}^{\alpha}(t) .
$$

Donc $h_{0} \in H_{2}$. Soit $P_{H_{2}}$ la projection orthogonale de $H$ sur $H_{2}$. Notons $H_{1}=P_{H_{2}}^{-1}(0)$, c'est-à-dire:

$$
H_{1}=\left\{v \in H \mid \sum_{i=1}^{m} \int_{0}^{1} Z_{1}\left(h_{0}\right) Z_{t}^{-1}\left(h_{0}\right) A_{i}\left(\xi_{t}\left(h_{0}\right)\right) \dot{v}^{i}(t) d t=0\right\} .
$$

On a: $H=H_{1} \oplus H_{2}$.

Soit $Y$ la fermeture de $H_{1}$ dans $X$, alors:

$$
Y=\left\{x \in X \mid\left\langle e_{i}, x\right\rangle=0, i=1, \ldots, d\right\}
$$

et $X=Y \oplus H_{2}$, le couple $\left(Y, H_{1}\right)$ est un espace de Wiener abstrait. Soit $\mu_{Y}$ la mesure de Wiener sur $Y$, on a: $\mu=\mu_{Y} \otimes \mu_{H_{2}}$, où $\mu_{H_{2}}$ est la mesure gaussienne canonique sur $\mathrm{H}_{2}$.

Notons $\Pi_{Y}$ la projection de $X$ sur $Y, \Pi_{H_{2}}$ la projection de $X$ sur $H_{2}$. Posons:

$$
\begin{aligned}
& \Omega_{n, R, R^{\prime}}^{1}=\Pi_{Y}\left(G_{n, R, R^{\prime}} \cap \Pi_{H_{2}}^{-1}\left(B_{H_{2}}\left(0, R^{\prime}\right)\right)\right), \\
& \Omega_{n, R, R^{\prime}}^{2}=\Pi_{Y}\left(Q_{n, R, R^{\prime}} \cap \Pi_{H_{2}}^{-1}\left(B_{H_{2}}\left(0, R^{\prime}\right)\right)\right) .
\end{aligned}
$$

Proposition 3.1. Notons $\Omega_{n, R, R^{\prime}}^{1, c}$ le complémentaire de $\Omega_{n, R, R^{\prime}}^{1}$ dans $Y$, soit $y \in \Omega_{n, R, R^{\prime}}^{1, c}$ alors: $\varepsilon y \in \Omega_{n, R, R^{\prime}}^{2, c}$ pour tout $\varepsilon \in[0,1]$.

Démonstration. Soit $y \in \Omega_{n, R, R^{\prime}}^{1, c}$, on a alors: $(y, z) \in G_{n, R, R^{\prime}}^{c}$ pour tout $z \in B_{H_{2}}\left(0, R^{\prime}\right)$, en particulier $(y, 0) \in G_{n, R, R^{\prime}}^{c}$. D'après le Corollaire 2.9: pour 
tout $\varepsilon \in[0,1],(\varepsilon y, 0) \in G_{n, R, R^{\prime}}^{c}$ et $(\varepsilon y, z) \in Q_{n, R, R^{\prime}}^{c}$ si $z \in B_{H_{2}}\left(0, R^{\prime}\right)$, par conséquent: $\varepsilon y \in \Omega_{n, R, R^{\prime}}^{2, c}$.

Estimation de terme résiduel. Soit $\delta>0$, soit $\widetilde{G}_{\delta} \in \mathscr{C}_{c}^{\infty}\left(H_{2}, \mathbb{R}\right)$ telle que:

$$
\widetilde{G}_{\delta}(z)= \begin{cases}1 & \text { si } z \in \overline{B_{H_{2}}\left(h_{0}, \delta / 2\right)}, \quad 0 \leq \widetilde{G}_{\delta} \leq 1 . \\ 0 & \text { si } z \notin B_{H_{2}}\left(h_{0}, \delta\right),\end{cases}
$$

Notons $G_{\delta}(x)=\widetilde{G}_{\delta}\left(\Pi_{H_{2}}(x)\right)$, et $G_{\delta, \varepsilon}=G_{\delta}(\varepsilon x)$, il est clair que:

$$
G_{\delta, \varepsilon} \in W_{\infty}(\mathbb{R}) \text { et } \sup _{\varepsilon \leq 1} E\left(\left\|\nabla^{k} G_{\delta, \varepsilon}\right\|^{p}\right)<+\infty .
$$

Proposition 3.2. Soit $\delta>0$, il existe $c>0$ telle que:

$$
\int_{\varphi_{\varepsilon}^{-1}(\xi)}\left(1-G_{\delta, \varepsilon}\right) \frac{d \mathscr{H}^{\infty-d}}{\operatorname{det} \varphi_{\varepsilon}}=O\left(\exp -\frac{1}{2 \varepsilon^{2}}\left(\left\|h_{0}\right\|_{H}^{2}+c\right)\right) \quad(\varepsilon \rightarrow 0) .
$$

Démonstration. Soit $\theta \in \mathscr{C}_{c}^{\infty}(\mathbb{R}, \mathbb{R})$ telle que $0 \leq \theta(t) \leq 1$ et

$$
\theta(t)= \begin{cases}1 & \text { si }|t| \leq 1 / 2 \\ 0 & \text { si }|t|>1\end{cases}
$$

On a, pour tout $u>0$ :

$$
\begin{aligned}
\int_{\varphi_{\varepsilon}^{-1}(\xi)}\left(1-G_{\delta, \varepsilon}\right) \frac{d \mathscr{H}^{\infty-d}}{\operatorname{det} \varphi_{\varepsilon}} & =\int_{\varphi_{\varepsilon}^{-1}(\xi)}\left(1-G_{\delta, \varepsilon}\right) \theta\left(\frac{\left|\varphi_{\varepsilon}-\xi\right|^{2}}{u^{2}}\right) \frac{d \mathscr{H}^{\infty-d}}{\operatorname{det} \varphi_{\varepsilon}} \\
& =\left\langle\delta_{\xi}\left(\varphi_{\varepsilon}\right),\left(1-G_{\delta, \varepsilon}\right) \theta\left(\frac{\left|\varphi_{\varepsilon}-\xi\right|^{2}}{u^{2}}\right)\right\rangle
\end{aligned}
$$

où $\delta_{\xi}$ est la mesure de Dirac au point $\xi$. (Voir [A-M, WA $\mathrm{WA}_{1}$.)

En utilisant l'intégration par partie comme [WA $\left.{ }_{2}\right]$, il vient:

$$
\begin{aligned}
\int_{\varphi_{\varepsilon}^{-1}(\xi)} & \left(1-G_{\delta, \varepsilon}\right) \frac{d \mathscr{H}^{\infty-d}}{\operatorname{det} \varphi_{\varepsilon}} \\
= & \sum_{\alpha, k, l, j} \int F_{k}(\varepsilon, x) \theta^{(l)}\left(\frac{\left|\varphi_{\varepsilon}-\xi\right|^{2}}{u^{2}}\right) C_{j}\left(\varphi_{\varepsilon}\right) Q_{\alpha}(x) d \mu(x)
\end{aligned}
$$

où la sommation est de termes finis, $F(\varepsilon, x)$ est un polynôme en $\varphi_{\varepsilon}(x)$, des dérivés de $\varphi_{\varepsilon}(x), \gamma_{\varepsilon}(x)=\sigma_{\varepsilon}^{-1}(x) \quad\left(\sigma_{\varepsilon}\right.$ étant la matrice de covariance de Malliavin de $\left.\varphi_{\varepsilon}\right) ; C_{j}$ sont des fonctions bornées sur $\mathbb{R}^{d}, Q_{0}$ est un polynôme en $1-G_{\delta, \varepsilon}$ avec $Q_{0}(0)=0, Q_{\alpha}\left(G_{\varepsilon}\right) \quad(\alpha \geq 1)$ est un polynôme en dérivées de $G_{\delta, \varepsilon}$ avec $Q_{\alpha}(0)=0$. D'après [K-S]: il existe $n_{0}>0$, tel que, pour tout $p>1$, on ait:

$$
E\left(\left|\operatorname{det} \sigma_{\varepsilon}^{-1}\right|^{p}\right) \leq c \varepsilon^{-n_{0}}
$$

donc il existe $N>0$ tel que

$$
E\left(\left|F_{k}(\varepsilon, x)\right|^{p}\right)^{1 / p}=O\left(\varepsilon^{-N}\right) .
$$

Compte tenu de (3.8), on en déduit:

$$
\int_{\varphi_{\varepsilon}^{-1}(\xi)}\left(1-G_{\delta, \varepsilon}\right) \frac{d \mathscr{C}^{\infty-d}}{\operatorname{det} \varphi_{\varepsilon}} \leq K \varepsilon^{-N} \mu\left(Y \times B_{H_{2}}^{c}\left(\frac{h_{0}}{\varepsilon}, \frac{\delta}{2 \varepsilon}\right) \cap\left\{\left|\varphi_{\varepsilon}-\xi\right| \leq u\right\}\right)^{1 / q}
$$

où $1 / p+1 / q=1$. 
Donc:

$$
\begin{aligned}
\varlimsup_{\varepsilon \rightarrow 0} 2 \varepsilon^{2} \log \int_{\varphi_{\varepsilon}^{-1}(\xi)}\left(1-G_{\delta, \varepsilon}\right) \frac{d \mathscr{H}^{\infty-d}}{\operatorname{det} \varphi_{\varepsilon}} \\
\leq \frac{1}{q} \varlimsup_{\varepsilon \rightarrow 0} 2 \varepsilon^{2} \log \mu\left(Y \times B_{H_{2}}^{c}\left(\frac{h_{0}}{\varepsilon}, \frac{\delta}{2 \varepsilon}\right) \cap\left\{\left|\varphi_{\varepsilon}-\xi\right| \leq u\right\}\right) .
\end{aligned}
$$

Remarquons que $q>1$ peut être choisi arbitraiement, on a ainsi:

$$
\begin{aligned}
\varlimsup_{\varepsilon \rightarrow 0} 2 & \varepsilon^{2} \log \int_{\varphi_{\varepsilon}^{-1}(\xi)}\left(1-G_{\delta, \varepsilon}\right) \frac{d \mathscr{H}^{\infty-d}}{\operatorname{det} \varphi_{\varepsilon}} \\
\leq & \varlimsup_{\varepsilon \rightarrow 0} 2 \varepsilon^{2} \log \mu\left(Y \times B_{H_{2}}^{c}\left(\frac{h_{0}}{\varepsilon}, \frac{\delta}{2 \varepsilon}\right) \cap\left\{\left|\varphi_{\varepsilon}-\xi\right| \leq u\right\}\right) \\
\leq & -\inf \left\{\|h\|_{H}^{2} \mid h \in Y \times B_{H_{2}}^{c}\left(h_{0}, \frac{\delta}{2}\right) \cap\{|\varphi-\xi| \leq u\}\right\} .
\end{aligned}
$$

Ici, on a utilisé la théorie de grandes déviations pour la diffusion de $\left[\mathrm{AZ}_{1}\right]$ ou [ST].

On a, pour certain $u>0$,

$$
\inf \left\{\|h\|_{H}^{2} \mid h \in Y \times B_{H_{2}}^{c}\left(h_{0}, \delta / 2\right) \cap\{|\varphi-\xi| \leq u\}\right\}>\left\|h_{0}\right\|_{H}^{2} .
$$

En effet, si (3.10) était faux pour tout $u>0$, en prenant $u=1 / n$, il existerait $h_{n} \in Y \times B_{H_{2}}^{c}\left(h_{0}, \delta / 2\right),\left|\varphi\left(h_{n}\right)-\xi\right| \leq 1 / n$ tels que $\left\|h_{n}\right\|_{H} \leq\left\|h_{0}\right\|_{H}$, donc il existerait une sous suite notée encore $h_{n}$ qui converge faiblement vers $h^{\prime}$, on a $\left\|h_{n}-h^{\prime}\right\|_{\infty} \rightarrow 0$, donc $h^{\prime} \in Y \times B_{H_{2}}^{c}\left(h_{0}, \delta / 2\right)$. D'autre part:

$$
\left|\varphi\left(h^{\prime}\right)-\xi\right|=\lim _{n \rightarrow \infty}\left|\varphi\left(h_{n}\right)-\xi\right| \leq \lim _{n \rightarrow \infty} \frac{1}{n}=0,
$$

ce qui implique que $h^{\prime} \in K_{x}^{\xi}$. D'après l'hypothèse (H.2), on a $h_{0}=h^{\prime}$, ce qui est en contradiction avec $h^{\prime} \in Y \times B_{H_{2}}^{c}\left(h_{0}, \delta / 2\right)$.

D'après (3.10), il existe $c>0$ tel que

$$
\inf \left\{\left\|h_{0}\right\|_{H}^{2} \mid h \in Y \times B_{H_{2}}^{c}\left(h_{0}, \delta / 2\right) \cap\{|\varphi-\xi| \leq u\}\right\} \geq\left\|h_{0}\right\|_{H}^{2}+c .
$$

On obtient donc le résultat.

Soit $y \in \Omega_{n, R, R^{\prime}}^{1, c}$, d'après le Lemme 3.1, pour tout $\alpha \in[0,1], z \in B_{H_{2}}\left(0, R^{\prime}\right)$. On a: $(\alpha y, z) \in Q_{n, R, R^{\prime}}^{c}$. Posons:

$$
\begin{aligned}
J_{\varepsilon}(\alpha, z, y)=\int_{0}^{1}( & \left|Z_{t}^{-1}(\varepsilon \alpha y, z)-Z_{t}^{-1}(z)\right|^{2} \\
+ & \left|\varphi_{t}(\varepsilon \alpha y, z)-\varphi_{t}(z)\right|^{2} \\
+\left|Z_{1}(\varepsilon \alpha y, z)-Z_{1}(z)\right|^{2} & +\left|\partial_{z} Z_{t}^{-1}(\varepsilon \alpha y, z)-\partial_{z} Z_{t}^{-1}(z)\right|^{2} \\
& \left.+\left|\partial_{z} Z_{1}(\varepsilon \alpha y, z)-\partial_{z} Z_{1}(z)\right|^{2}\right) d i
\end{aligned}
$$

et

$$
I_{\varepsilon, r}(y)=\int_{0}^{1} \int_{B_{H_{2}}\left(h_{0}, r\right)}\left[\left|J_{\varepsilon}(\alpha, z, y)\right|^{k}+\left|\partial_{\alpha} J_{\varepsilon}(\alpha, z, y)\right|^{k}+\left|\partial_{z} J_{\varepsilon}(\alpha, z, y)\right|^{k}\right] d \alpha d z
$$

D'après un lemme de Sobolev, il existe une constante $a>0$ telle que:

$$
\sup _{0 \leq \alpha \leq 1} \sup _{z \in B_{H_{2}}\left(h_{0}, r\right)} J_{\varepsilon}(\alpha, z, y) \leq a I_{\varepsilon, r}(y)
$$

pour un certain $k>d+1$. 
Soit $\rho>0$, soit $\kappa_{\rho} \in \mathscr{C}_{c}^{\infty}(\mathbb{R}, \mathbb{R}), 0 \leq \kappa_{\rho}(t) \leq 1$ telle que:

$$
\kappa_{\rho}(t)= \begin{cases}1 & \text { si } t<\rho / 2 a \\ 0 & \text { si } t \geq \rho / a\end{cases}
$$

Notons $\widetilde{F}_{\rho, \varepsilon, r}(y)=\kappa_{\rho}\left(I_{\varepsilon, r}(y)\right)$ et $F_{\rho, \varepsilon, r}(x)=\widetilde{F}_{\rho, \varepsilon, r}\left(\Pi_{Y}(x)\right)$.

Lemme 3.3. (i) $G_{\delta, \varepsilon} F_{\rho, \varepsilon, r} \in W_{\infty}(\mathbb{R})$;

(ii) $\sup _{\varepsilon \leq 1} E\left(\left\|\nabla^{m}\left(G_{\delta, \varepsilon} F_{\rho, \varepsilon, r}\right)\right\|_{\otimes^{m} H}^{p}\right)<+\infty$.

Démonstration. On a:

$$
G_{\delta, \varepsilon} \varphi_{t}(\alpha y, z)=G_{\delta, \varepsilon} \xi_{\alpha, z-\alpha \pi_{H_{2}}(x)}(t, x) \quad \mu \text {-p.p, }
$$

d'après le Lemme 2.3 de [O-P], on voit que $x \rightarrow \xi_{\alpha, z-\alpha \pi_{H_{2}}(x)}(t, x)$ est un élément de $W_{\infty}\left(\mathbb{R}^{d}\right)$, par suite $G_{\delta, \varepsilon} \varphi_{t}(\alpha y, z) \in W_{\infty}\left(\mathbb{R}^{d}\right)$. De même pour $Z_{t}^{-1}(\alpha y, z)$. (i) se voit donc par leurs définitions; (ii) résulte de l'estimation:

$$
\sup _{\varepsilon \leq 1} \sup _{\|z\| \leq R^{\prime}} E\left(\sup _{t \leq 1}\left\|\partial_{H_{2}}^{m} \partial_{\xi_{0}}^{m^{\prime}} \xi_{\varepsilon, z}(t)\right\|^{p}\right) \leq C_{m, m^{\prime}}<+\infty .
$$

Proposition 3.4. Il existe une constante $c>0$ telle que:

$$
\int_{\varphi_{\varepsilon}^{-1}(\xi)}\left(1-F_{\rho, \varepsilon, r}\right) G_{\delta, \varepsilon} \frac{d \mathscr{H}^{\infty}-d}{\operatorname{det} \varphi_{\varepsilon}}=O\left(\exp -\frac{1}{2 \varepsilon^{2}}\left(\left\|h_{0}\right\|_{H}^{2}+c\right)\right) \quad(\varepsilon \rightarrow 0) .
$$

Démonstration. D'après le Lemme 3.3 et les démarches de la démonstration de la Proposition 3.2, (3.12) sera établi dès qu'on aura démontré le résultat suivant dont la démonstration sera remise en appendice.

Lemme 3.5. Pour tout $\rho>0$ et $\sigma>0$ :

$$
\begin{gathered}
\varlimsup_{\varepsilon \rightarrow 0} 2 \varepsilon^{2} \log \mu\left(\left(\left\{I_{\varepsilon, r}(y) \geq \rho\right\} \times \varepsilon^{-1} B_{H_{2}}\left(h_{0}, \delta\right)\right) \cap\{|\varphi(\varepsilon x)-\xi| \leq \sigma\}\right) \\
\leq-\inf \left\{\|h\|_{H}^{2}|| \varphi(h)-\xi \mid \leq \sigma, I_{1, r}\left(P_{H_{1}}(h)\right) \geq \rho\right\} .
\end{gathered}
$$

D'après les Propositions 3.2 et 3.4 , la contribution principale à $p\left(\varepsilon, \xi_{0}, \xi\right)$ provient de $\int_{\varphi_{\varepsilon}^{-1}(\xi)} F_{\rho, \varepsilon, r} G_{\delta, \varepsilon} d \mathscr{H}^{\infty-d} / \operatorname{det} \varphi_{\varepsilon}$.

Estimation du terme principal. Désignons par $\mathscr{E}\left(B_{H_{2}}\left(0, R^{\prime}\right)\right)$ l'espace des fonctions indéfiniment dérivables sur $B_{H_{2}}\left(0, R^{\prime}\right)$, munie de la topologie Fréchet usuelle de la convergence uniforme sur tout compact de $B_{H_{2}}\left(0, R^{\prime}\right)$. D'après "le splitting lemma" de [MA 2 , l'ensemble $\Xi_{R, R^{\prime}}^{1}=\bigcap_{n} \Omega_{n, R, R^{\prime}}^{1}, \Xi_{R, R^{\prime}}^{2}=$ $\bigcap_{n} \Omega_{n, R, R^{\prime}}^{2}$ sont des ensembles minces de $Y$. Pour tout $y \in \Omega_{n, R, R^{\prime}}^{2, c}$, définissons la fonction partielle $\varphi_{y}(z): B_{H_{2}}\left(0, R^{\prime}\right) \rightarrow \mathbb{R}^{d}$ par $\varphi_{y}(z)=\varphi(y, z)$, qui définit une application: $\Omega_{n, R, R^{\prime}}^{2, c} \rightarrow \mathscr{E}\left(B_{H_{2}}\left(0, R^{\prime} / 2\right)\right), y \rightarrow \varphi_{y}$ continue.

D'après la construction de $F_{\rho, \varepsilon, r}$, il existe $\rho>0$ assez petit tel que pour tout $y \notin F_{\rho, \varepsilon, r}^{-1}(\{0\}), \varphi_{\varepsilon y}$ définisse un difféomorphisme de la boule $B_{H_{2}}\left(h_{0}, \rho\right)$ sur un voisinage ouvert de $\xi$, notons $p_{\varepsilon}(y)(\cdot)$ l'application inverse de $\varphi_{\varepsilon y}$ :

$$
\varphi\left(\varepsilon y, p_{\varepsilon}(y)(\eta)\right)=\eta \quad \text { sur un voisinage de } \xi .
$$

Soit $r>0$ tel que:

$$
\left\{z \in B_{H_{2}}\left(h_{0}, r\right), \varphi(0, z)=\xi\right\}=\left\{h_{0}\right\}, \quad R^{\prime}>2\left\|h_{0}\right\|_{H}, R \geq 2 R^{\prime} .
$$


Proposition 3.6. On a:

$$
\begin{aligned}
& \int_{\varphi_{\varepsilon}^{-1}(\xi)} F_{\rho, \varepsilon, r} G_{\delta, \varepsilon} \frac{d \mathscr{H}^{\infty}-d}{\operatorname{det} \varphi_{\varepsilon}}=\frac{\exp -d^{2}\left(\xi_{0}, \xi\right) / 2 \varepsilon^{2}}{\left(2 \pi \varepsilon^{2}\right)^{d / 2}} \\
& \times \int_{Y} \widetilde{F}_{\rho, \varepsilon, r}(y)\left[\exp -\frac{1}{2 \varepsilon^{2}}\left(\left|p_{\varepsilon}(y)(\xi)-h_{0}\right|^{2}\right.\right. \\
&\left.\left.+2\left\langle p_{\varepsilon}(y)(\xi)-h_{0}, h_{0}\right\rangle\right)\right] J p_{\varepsilon}(y)(\xi) d \mu_{Y}
\end{aligned}
$$

où $J p_{\varepsilon}(y)$ désigne le jacobien de $p_{\varepsilon}(y)$.

Démonstration. On a:

$$
\begin{aligned}
\int_{\varphi_{\varepsilon}^{-1}(\xi)} & F_{\rho, \varepsilon, r} G_{\delta, \varepsilon} \frac{d \mathscr{C}^{\infty-d}}{\operatorname{det} \varphi_{\varepsilon}}=\lim _{\sigma \rightarrow 0} \frac{1}{\operatorname{vol}(B(\xi, \sigma))} \int F_{\rho, \varepsilon, r} G_{\delta, \varepsilon} \mathbf{1}_{\left|\varphi_{\varepsilon}-\xi\right| \leq \sigma} d \mu(x) \\
= & \lim _{\sigma \rightarrow 0} \frac{1}{\operatorname{vol}(B(\xi, \sigma))} \int \widetilde{F}_{\rho, \varepsilon, r}(y) \widetilde{G}_{\delta, \varepsilon}(z) \mathbf{1}_{\left|\varphi_{\varepsilon}-\xi\right| \leq \sigma} d \mu_{Y}(y) d \mu_{H_{2}}(z) \\
= & \lim _{\sigma \rightarrow 0} \frac{1}{\operatorname{vol}(B(\xi, \sigma))} \times a_{\sigma}
\end{aligned}
$$

où

$$
\begin{aligned}
a_{\alpha} & =\int \widetilde{F}_{\rho, \varepsilon, r}(y) \widetilde{G}_{\delta, \varepsilon}(z) \mathbf{1}_{\left|\varphi_{\varepsilon}-\xi\right| \leq \sigma} d \mu_{Y}(y) d \mu_{H_{2}}(z) \\
& =\int_{Y} \widetilde{F}_{\rho, \varepsilon, r}(y) d \mu_{Y}(y) \int_{H_{2}} \widetilde{G}_{\delta}(z) \mathbf{1}_{|\varphi-\xi| \leq \sigma}(\varepsilon y, z) \frac{\exp -|z|^{2} / 2 \varepsilon^{2}}{\left(2 \pi \varepsilon^{2}\right)^{d / 2}} d z .
\end{aligned}
$$

En faisant le changement de variable $z=p_{\varepsilon}(y)(\eta)$ sur un voisinage de $z \in$ $B_{H_{2}}\left(h_{0}, \rho\right) \cap \varphi_{\varepsilon y}^{-1}(\{\xi\})$ dans (3.15), on obtient:

$$
\lim _{\sigma \rightarrow 0} \frac{1}{\operatorname{vol}(B(\xi, \sigma))} \times a_{\sigma}=\int_{Y} \widetilde{F}_{\rho, \varepsilon, r}(y)\left[\exp -\frac{1}{2 \varepsilon^{2}}\left|p_{\varepsilon}(y)(\xi)\right|^{2}\right] J p_{\varepsilon}(y)(\xi) d \mu_{Y}(y)
$$

d'où (3.14).

Dans ce qui suit, on va supposer que les deux points $\xi_{0}, \xi$ sont non conjugués le long de l'unique géodésique $h_{0}$ reliant $\xi_{0}$ et $\xi$, ce qui se formule par l'hypothèse suivante:

(H.3) $h_{0}$ est un minimum non dégénéré de l'application $h \rightarrow \frac{1}{2}\|h\|_{H}^{2}$ sur la sous variété $K_{\xi_{0}}^{\xi}$ de $H$.

Pour alléger les notations, on notera $p_{\varepsilon}(y)$ à la place de $p_{\varepsilon}(y)(\xi), p_{\varepsilon}^{\prime}(y)$ la dérivée de $p_{\varepsilon}(y)$ par rapport à $\varepsilon$ et $\widetilde{F}_{\rho, \varepsilon}$ à la place de $\widetilde{F}_{\rho, \varepsilon, r}$.

D'après (3.14), il suffit d'estimer le terme suivant: pour tout $p>1$, il existe $\rho>0, \varepsilon_{0}>0$ tels que:

$$
\sup _{\varepsilon \leq \varepsilon_{0}} \int \widetilde{F}_{\rho, \varepsilon}(y) \exp \left[\frac{p}{\varepsilon^{2}}\left\langle h_{0}, p_{\varepsilon}(y)-h_{0}\right\rangle\right] d \mu_{Y}(y)<+\infty .
$$

Nous avons:

$$
p_{\varepsilon}(y)=h_{0}+\int_{0}^{\varepsilon} p_{\tau}^{\prime}(y) d \tau
$$

et

$$
p_{\varepsilon}^{\prime}(y)=-\partial_{H_{2}}^{-1} \varphi\left(\varepsilon y, p_{\varepsilon}(y)\right) \cdot \partial_{\varepsilon} \varphi\left(\varepsilon y, p_{\varepsilon}(y)\right)
$$


On voit clairement que $\partial_{\varepsilon} \varphi\left(\varepsilon y, p_{\varepsilon}(y)\right)_{\mid \varepsilon=0}=\left\langle\nabla \varphi\left(h_{0}\right), y\right\rangle=0$, par suite:

$$
\begin{aligned}
\partial_{\varepsilon} \varphi\left(\varepsilon y, p_{\varepsilon}(y)\right)= & \int_{0}^{\varepsilon}\left[\partial_{s}^{2} \varphi\left(s y, p_{s}(y)\right)+\partial_{s} \partial_{H_{2}} \varphi\left(s y, p_{s}(y)\right) p_{s}^{\prime}(y)\right] d s \\
= & \int_{0}^{\varepsilon}\left[\partial_{s}^{2} \varphi\left(s y, p_{s}(y)\right)\right] d s \\
& -\int_{0}^{\varepsilon}\left[\partial_{s} \partial_{H_{2}} \varphi\left(s y, p_{s}(y)\right) \partial_{H_{2}}^{-1} \varphi\left(s y, p_{s}(y)\right) \partial_{s} \varphi\left(s y, p_{s}(y)\right)\right] d s \\
= & I_{1}(\varepsilon, y)+I_{2}(\varepsilon, y) .
\end{aligned}
$$

Soit $\gamma_{t}$ la solution de l'équation différentielle stochastique suivante:

$$
\begin{aligned}
\gamma_{t}= & \int_{0}^{t} \partial A_{i}\left(\varphi_{s}(z)\right) \eta_{s}(z) d x^{i}(s)+\int_{0}^{t} A_{0}\left(\varphi_{s}(z)\right) d s \\
& +\int_{0}^{t} \partial A_{i}\left(\varphi_{s}(z)\right) \gamma_{s} \dot{z}_{s}^{i} d s+\frac{1}{2} \int_{0}^{t} \partial^{2} A_{i}\left(\varphi_{s}(z)\right) \cdot \eta_{s}(z) \cdot \eta_{s}(z) d s
\end{aligned}
$$

avec

$$
A_{0}=\frac{1}{2} \sum_{i, k} \partial_{i} A_{k} A_{k}^{i}
$$

et

$$
\eta_{t}(z)=\int_{0}^{t} Z_{t}(z) Z_{s}^{-1}(z) A_{i}\left(\varphi_{s}(z)\right) d x^{i}(s) .
$$

Alors $\partial_{\varepsilon}^{2} \varphi(\varepsilon x+z)_{\mid \varepsilon=0}=\gamma_{1}(x, z)$.

Lemme 3.7. Notons:

$$
\begin{aligned}
& J_{1}(y)=\int_{0}^{\varepsilon} \partial_{H_{2}}^{-1} \varphi\left(\tau y, p_{\tau}(y)\right)\left[I_{1}(\tau, y)-\tau \gamma_{1}\left(y, h_{0}\right)\right] d \tau, \\
& J_{2}(y)=\int_{0}^{\varepsilon} \partial_{H_{2}}^{-1} \varphi\left(\tau y, p_{\tau}(y)\right) I_{2}(\tau, y) d \tau .
\end{aligned}
$$

Alors pour tout $p>1$, il existe $\rho>0, \varepsilon_{0}>0$ tels que:

$$
\begin{aligned}
& \sup _{\varepsilon \leq \varepsilon_{0}} \int_{Y} \widetilde{F}_{\rho, \varepsilon}(y) \exp \left[-\frac{p}{\varepsilon^{2}}\left\langle h_{0}, J_{1}(y)\right\rangle\right] d \mu_{Y}(y)<+\infty, \\
& \sup _{\varepsilon \leq \varepsilon_{0}} \int_{Y} \widetilde{F}_{\rho, \varepsilon}(y) \exp \left[-\frac{p}{\varepsilon^{2}}\left\langle h_{0}, J_{2}(y)\right\rangle\right] d \mu_{Y}(y)<+\infty .
\end{aligned}
$$

Démonstration. Nous montrons ici (3.21), et (3.20) se démontre de la même manière comme dans $\left[\mathrm{WA}_{2}\right]$.

Nous avons: $\exp \left[-\left(p / \varepsilon^{2}\right)\left\langle h_{0}, J_{2}(y)\right\rangle\right] \leq \exp \left(p / \varepsilon^{2}\right)\left|h_{0}\right|\left|J_{2}(y)\right|$; pour $y$ tel que $\widetilde{F}_{\rho, \varepsilon}(y) \neq 0$, on a: $\sup _{\tau \leq \varepsilon}\left|\partial_{H_{2}}^{-1} \varphi\left(\tau y, p_{\tau}(y)\right)\right|<M$, où $M$ est une constante indépendante de $y$. Donc il suffit de montrer:

(3.22) il existe $\rho>0, \varepsilon_{0}>0$ tels que: pour tout $\varepsilon \leq \varepsilon_{0}$ :

$$
\int_{Y} \widetilde{F}_{\rho, \varepsilon}(y) \exp p\left|\partial_{\varepsilon} \partial_{H_{2}} \varphi\left(\varepsilon y, p_{\varepsilon}(y)\right)\right|\left|\partial_{\varepsilon} \varphi\left(\varepsilon y, p_{\varepsilon}(y)\right)\right| d \mu_{Y}(y)<+\infty .
$$

Considérons la fonction $N \in \mathscr{C}^{\infty}\left(\mathbb{R}^{d}, \mathbb{R}\right)$ telle que:

$(*) \quad N(\xi)=|\xi|$ en dehors d'un voisinage compact de 0 et $N(\xi) \geq|\xi|$. 
Nous avons:

$$
\begin{aligned}
\exp p & \left|\partial_{\varepsilon} \partial_{H_{2}} \varphi\left(\varepsilon y, p_{\varepsilon}(y)\right)\right|\left|\partial_{\varepsilon} \varphi\left(\varepsilon y, p_{\varepsilon}(y)\right)\right| \\
& =\exp p\left|\partial_{\varepsilon} \partial_{H_{2}} \varphi\left(\varepsilon y, p_{\varepsilon}(y)\right)\right|\left|\partial_{\varepsilon} \varphi\left(\varepsilon y, p_{\varepsilon}(y)\right)-\left\langle\nabla \varphi\left(h_{0}\right), y\right\rangle\right| \\
& \leq \exp p N\left(\partial_{\varepsilon} \partial_{H_{2}} \varphi\left(\varepsilon y, p_{\varepsilon}(y)\right)\right) \cdot N\left(\partial_{\varepsilon} \varphi\left(\varepsilon y, p_{\varepsilon}(y)\right)-\left\langle\nabla \varphi\left(h_{0}\right), y\right\rangle\right) \\
& \leq\left\|\exp p N\left(\partial_{\varepsilon} \partial_{H_{2}} \varphi(\varepsilon y, z)\right) \cdot N\left(\partial_{\varepsilon} \varphi(\varepsilon y, z)-\left\langle\nabla \varphi\left(h_{0}\right), y\right\rangle\right)\right\|_{z, \rho}
\end{aligned}
$$

où

$$
\|f(z)\|_{z, \rho}=\sup _{z \in B_{H_{2}}\left(h_{0}, \rho\right)}|f(z)|
$$

\section{Notons}

$$
\begin{aligned}
b(y)= & \left(\int_{B_{H_{2}}\left(h_{0}, \rho\right)}\left[\exp p k N\left(\partial_{\varepsilon} \partial_{H_{2}} \varphi(\varepsilon y, z)\right) N\left(\partial_{\varepsilon} \varphi(\varepsilon y, z)-\left\langle\nabla \varphi\left(h_{0}\right), y\right\rangle\right)\right]\right. \\
& \left.\times\left[1+\left|\partial_{z} N\left(\partial_{\varepsilon} \partial_{H_{2}} \varphi(\varepsilon y, z)\right) \cdot N\left(\partial_{\varepsilon} \varphi(\varepsilon y, z)-\left\langle\nabla \varphi\left(h_{0}\right), y\right\rangle\right)\right|^{k}\right] d z\right)^{1 / k} .
\end{aligned}
$$

Alors on a:

$$
\exp p\left|\partial_{\varepsilon} \partial_{H_{2}} \varphi\left(\varepsilon y, p_{\varepsilon}(y)\right)\right|\left|\partial_{\varepsilon} \varphi\left(\varepsilon y, p_{\varepsilon}(y)\right)\right| \leq c b(y)
$$

Donc:

$$
\begin{aligned}
& \int_{Y} \widetilde{F}_{\rho, \varepsilon}(y) \exp \left[p\left|\partial_{\varepsilon} \partial_{H_{2}} \varphi\left(\varepsilon y, p_{\varepsilon}(y)\right)\right|\left|\partial_{\varepsilon} \varphi\left(\varepsilon y, p_{\varepsilon}(y)\right)\right|\right] d \mu_{Y}(y) \\
& \quad \leq c \int_{Y} \widetilde{F}_{\rho, \varepsilon}(y) b(y) d \mu_{Y}(y)=c E\left(\widetilde{F}_{\rho, \varepsilon}(x) b(x) \mid \Pi_{H_{2}}(x)=0\right)
\end{aligned}
$$

où $\widetilde{F}_{\rho, \varepsilon}(x)$ (resp. $b(x)$ ) est obtenu en substituant $y$ par $x$ dans la définition de $\widetilde{F}_{\rho, \varepsilon}(y)$, voir (3.11) (resp. $b(y)$, voir (3.23)).

$$
\leq c\left\|\widetilde{F}_{\rho, \varepsilon}(x) b(x)\right\|_{W_{2 r}^{q}}
$$

Comme dans [ $\mathrm{WA}_{2}$ ], on voit aisément que:

(3.25) Pour tout $p>1$, il existe $\rho>0, \varepsilon_{0}>0$ tels que pour tout $\varepsilon \leq \varepsilon_{0}$ :

$$
\begin{aligned}
E\left(\widetilde{F}_{\rho, \varepsilon}(x)\right. & \left.\int_{B_{H_{2}}\left(h_{0}, r\right)}\left[\exp p\left|\partial_{\varepsilon} \partial_{z} \varphi(\varepsilon x, z)\right|\left|\partial_{\varepsilon} \varphi(\varepsilon x, z)-\left\langle\nabla \varphi\left(h_{0}\right), x\right\rangle\right|\right] d z\right) \\
& <+\infty .
\end{aligned}
$$

On en déduit avec le Lemme 4 de [DO, p. 191]:

$$
\sup _{\varepsilon \leq \varepsilon_{0}}\left\|\widetilde{F}_{\rho, \varepsilon}(x) b(x)\right\|_{W_{2 r}^{q}}<+\infty \text { pour } r>d, q>1 \text {. }
$$

Donc:

$$
\left|E\left(\tilde{F}_{\rho, \varepsilon}(x) b(x) \mid \Pi_{H_{2}}(x)=0\right)\right| \leq C \sup _{\varepsilon \leq \varepsilon_{0}}\left\|\widetilde{F}_{\rho, \varepsilon}(x) b(x)\right\|_{W_{2 r}^{q}<+\infty} .
$$

On en déduit (3.22). 
Lemme 3.8. Sous l'hypothèse de non conjugaison (H.3), il existe $p>1, \rho>0$ tels que:

$$
\int_{Y} \widetilde{F}_{\rho, \varepsilon}(y) \exp \left[-\frac{p}{\varepsilon^{2}} \int_{0}^{\varepsilon}\left\langle h_{0}, \partial_{H_{2}}^{-1} \varphi\left(\tau y, p_{\tau}(y)\right) \cdot \tau \gamma_{1}\left(y, h_{0}\right)\right\rangle d \tau\right] d \mu_{Y}<+\infty .
$$

Démonstration. Sous l'hypothèse (H.3), on a (cf. $\left[\mathrm{BI}_{1}\right]$ ou $\left.[\mathrm{BE}]\right)$

$$
\int_{Y} \exp \left[-p\left\langle h_{0}, \partial_{H_{2}}^{-1} \varphi\left(h_{0}\right) \cdot \gamma_{1}\left(y, h_{0}\right)\right\rangle d \mu_{Y}<+\infty .\right.
$$

Par la construction de $F_{\rho, \varepsilon}$, si $\widetilde{F}_{\rho, \varepsilon}(y) \neq 0$, alors:

$$
\left\|\partial_{H_{2}} \varphi\left(\varepsilon y, p_{\varepsilon}(y)\right)-\partial_{H_{2}} \varphi\left(h_{0}\right)\right\| \leq \rho .
$$

Pour tout $\beta>0$ assez petit, il existe $\rho>0$ tel que:

$$
\left\|\partial_{H_{2}}^{-1} \varphi\left(\varepsilon y, p_{\varepsilon}(y)\right)-\partial_{H_{2}}^{-1} \varphi\left(h_{0}\right)\right\| \leq \beta .
$$

Il est clair que pour tout $q>1$, il existe $\beta>0$ assez petit tel que:

$$
E\left(\exp \beta q\left|\gamma_{1}\left(x, h_{0}\right)\right|\right)<+\infty
$$

ou encore:

$$
E\left(\exp \beta q N\left(\gamma_{1}\left(x, h_{0}\right)\right)\right)<+\infty .
$$

Donc:

$$
E\left(\exp \left[\beta N\left(\gamma_{1}\left(x, h_{0}\right)\right)\right] \mid \Pi_{H_{2}}(x)=0\right) \leq C \cdot\left\|\exp \left[\beta N\left(\gamma_{1}\left(x, h_{0}\right)\right)\right]\right\|_{W_{2 s}^{q}}<+\infty
$$

ou encore:

$$
\int_{Y} \exp \left[\beta N\left(\gamma_{1}\left(x, h_{0}\right)\right)\right] d \mu_{Y}(y)<+\infty .
$$

D'après (3.29), on a:

$\left\langle h_{0}, \partial_{H_{2}}^{-1} \varphi\left(\alpha y, p_{\alpha}(y)\right) \cdot \gamma_{1}\left(y, h_{0}\right)\right\rangle \leq\left\langle h_{0}, \partial_{H_{2}}^{-1} \varphi\left(h_{0}\right) \cdot \gamma_{1}\left(h_{0}\right)\right\rangle+\beta\left|h_{0}\right| N\left(\gamma_{1}\left(x, h_{0}\right)\right)$.

On établit donc le lemme avec (3.28) et (3.30).

\section{Développement asymptotique.}

Théorème. Sous les hypothèses (H.1), (H.2), et (H.3), pour tout entier $n>0$, on a, lorsque $\varepsilon \rightarrow 0$ :

$$
p_{\varepsilon}\left(\xi_{0}, \xi\right)=\frac{\exp -\left[d^{2}\left(\xi_{0}, \xi\right) / 2 \varepsilon\right]}{(\sqrt{2 \pi \varepsilon})^{d}\left[\operatorname{det} \varphi\left(h_{0}\right)\right]}\left(\sum_{k=0}^{n} c_{k} \varepsilon^{k}+o\left(\varepsilon^{n}\right)\right)
$$

avec

$$
c_{0}=\int_{Y} \exp -\frac{1}{2}\left\langle h_{0}, p(y)\right\rangle d \mu_{Y}(y) \text { où } p(y)=\left.\partial_{\varepsilon}^{2} p_{\varepsilon}(y)\right|_{\varepsilon=0} .
$$

Démonstration. Remarquons d'abord:

$$
\lim _{\varepsilon \rightarrow 0} J p_{\varepsilon y}(\xi)=J p_{0}(\xi)=\frac{1}{\operatorname{det}\left(\left(\nabla \varphi^{i}\left(h_{0}\right) \mid e_{j}\right)_{H}\right)}=\frac{1}{[\operatorname{det} \varphi]\left(h_{0}\right)} .
$$

et $\left.\partial_{\varepsilon}^{2} p_{\varepsilon}(y)\right|_{\varepsilon=0}=\partial_{H_{2}}^{-1} \varphi\left(h_{0}\right) \cdot \gamma_{1}\left(h_{0}\right)$. Dans (3.14), en faisant le développement limité sous le signe $\int$ et en procédant comme [ $\mathrm{BI}_{1}$, pp. 167-173], on obtient le résultat, le développement asymptotique sous le signe $\int$ est contrôlé par les Lemmes 3.7 et 3.8 . 


\section{APPENDICE}

L'objet de cette section est de démontrer le Lemme 3.5 admis:

Lemme 3.5. Pour tout $\rho>0, \sigma>0$ :

$$
\begin{gathered}
\varlimsup_{\varepsilon \rightarrow 0} 2 \varepsilon^{2} \log \mu\left(\left(\left\{I_{\varepsilon}(y) \geq \rho\right\} \times \varepsilon^{-1} B_{H_{2}}\left(h_{0}, \delta\right)\right) \cap\{|\varphi(\varepsilon x)-\xi| \leq \sigma\}\right) \\
\leq-\inf \left\{\|h\|_{H}^{2}|| \varphi(h)-\xi \mid \leq \sigma, I_{1}\left(P_{H_{1}}(h)\right) \geq \rho\right\} .
\end{gathered}
$$

La démonstration de ce résultat se base sur le théorie moderne de grande déviation pour les diffusions établie dans $\left[\mathrm{AZ}_{1}\right]$ out $[\mathrm{ST}]$. Dans ce qui suit, nous adoptons le schéma de [ST].

Soit $\varphi_{\varepsilon, t}^{n}(\alpha, z)$ la solution de l'équation différentielle stochastique suivante:

$$
\begin{aligned}
d \varphi_{\varepsilon, t}^{n}(\alpha, z) & =\varepsilon \alpha A_{i}\left(\varphi_{\varepsilon t_{n}}^{n}(\alpha z)\right) d x^{i}+\varepsilon^{2} \alpha^{2} A_{0}\left(\varphi_{\varepsilon, t}^{n}(\alpha, z)\right) d t+A_{i}\left(\varphi_{\varepsilon t}^{n}(\alpha, z)\right) d z^{i}, \\
\varphi_{\varepsilon, 0}^{n}(\alpha, z) & =\xi_{0},
\end{aligned}
$$

avec $t_{n}=[n t] / n$.

Lemme 4.1. Soit $\rho>0$, alors:

$$
\lim _{n \rightarrow+\infty} \varlimsup_{\varepsilon \rightarrow 0} \varepsilon^{2} \mu\left(x \in X \mid\left\|\varphi_{\varepsilon, t}^{n}(\alpha, z)-\varphi_{t}(\alpha \varepsilon x+z)\right\|_{t, \infty} \geq \rho\right)=-\infty
$$

uniformément en $(\alpha, z) \in[0,1] \times \overline{B_{H_{2}}\left(0, R^{\prime \prime}\right)}$. (4.1) est aussi valable pour les dérivées $\partial^{k} \varphi_{\varepsilon, t}^{n}(\alpha, z) \mid \partial \xi_{0}^{k}$, où $\|\cdot\|_{t, \infty}$ désigne la norme uniforme en $t$ sur $[0,1]$.

Démonstration. Voir [ST, Lemmes 4.7, 4.8, et 4.12].

Lemme 4.2. Soit $\rho>0$, on $a$ :

$$
\lim _{n \rightarrow+\infty} \varlimsup_{\varepsilon \rightarrow 0} \varepsilon^{2} \mu\left(x \in X \mid \sup _{(\alpha, z) \in K}\left\|\varphi_{\varepsilon, t}^{n}(\alpha, z)-\varphi_{t}(\alpha \varepsilon x+z)\right\|_{t, \infty} \geq \rho\right)=-\infty,
$$

de même pour les dérivées de $\varphi_{\varepsilon, t}^{n}(\alpha, z), \varphi_{t}(\alpha \varepsilon x+z)$ par rapport à $\xi_{0} \in \mathbb{R}^{d}$ où $K=[0,1] \times \overline{B_{H_{2}}\left(0, R^{\prime \prime}\right)}$.

Démonstration. Soit $\rho>0$ fixé, pour tout $L>0$, il existe $n_{L}>0$ tel que pour tout $n \geq n_{L}$, on ait:

$$
\varlimsup_{\varepsilon \rightarrow 0} \varepsilon^{2} \mu\left(x \in X \mid\left\|\varphi_{\varepsilon, t}^{n}(\alpha, z)-\varphi_{t}(\alpha \varepsilon x+z)\right\|_{t, \infty} \geq \rho / 2\right)<-L
$$

il existe $\varepsilon_{L}>0$ tel que pour tout $\varepsilon \leq \varepsilon_{L}$ :

$$
\mu\left(x \in X \mid\left\|\varphi_{\varepsilon, t}^{n}(\alpha, z)-\varphi_{t}(\alpha \varepsilon x+z)\right\|_{t, \infty} \geq \rho / 2\right) \leq e^{-L / \varepsilon^{2}} .
$$

Soit $l>0$, il existe $m=C \cdot[1 / l]^{d+1}$ points $\left(\alpha_{i}, z_{i}\right)$ de $K$ tels que:

$$
K \subset \bigcup_{i=1}^{m} B\left(\left(\alpha_{i}, z_{i}\right), l\right) .
$$

Notons:

$$
\begin{aligned}
f_{n}(x) & =\sup _{\varepsilon \leq 1} \sup _{(\alpha, z) \in K}\left\|\partial_{\alpha} \partial_{z} \varphi_{\varepsilon, t}^{n}(\alpha, z)\right\|_{t, \infty}, \\
f(x) & =\sup _{\varepsilon \leq 1} \sup _{(\alpha, z) \in K}\left\|\partial_{\alpha} \partial_{z} \varphi_{t}(\alpha \varepsilon x+z)\right\|_{t, \infty} .
\end{aligned}
$$


Il est facile de voir que:

$$
\sup _{n} E\left(f_{n}\right) \leq C<+\infty
$$

On a:

$$
\begin{aligned}
& \left\|\varphi_{\varepsilon, t}^{n}(\alpha, z)-\varphi_{\varepsilon, t}^{n}\left(\alpha^{\prime}, z^{\prime}\right)\right\|_{t, \infty} \\
& \quad \leq \int_{0}^{1}\left\|\partial_{\alpha} \partial_{z} \varphi_{\varepsilon, t}^{n}\left(u \alpha+(1-u) \alpha^{\prime}, u z+(1-u) z^{\prime}\right)\right\|_{t, \infty} d u\left(\left|\alpha-\alpha^{\prime}\right|+\left|z-z^{\prime}\right|\right) \\
& \quad \leq f_{n}(x)\left(\left|\alpha-\alpha^{\prime}\right|+\left|z-z^{\prime}\right|\right) .
\end{aligned}
$$

Soit $(\alpha, z) \in K$, il existe $i$ tel que $\left|\alpha-\alpha_{i}\right|+\left|z-z_{i}\right|<l$, on a:

$$
\begin{gathered}
\left\|\varphi_{\varepsilon, t}^{n}\left(\alpha_{i}, z_{i}\right)-\varphi_{t}\left(\alpha_{i} \varepsilon x+z_{i}\right)\right\|_{t, \infty} \geq\left\|\varphi_{t}(\alpha \varepsilon x+z)-\varphi_{\varepsilon, t}^{n}(\alpha, z)\right\|_{t, \infty} \\
\quad-\left\|\varphi_{\varepsilon, t}^{n}\left(\alpha_{i}, z_{i}\right)-\varphi_{\varepsilon, t}^{n}(\alpha, z)+\varphi_{t}(\alpha \varepsilon x+z)-\varphi_{t}\left(\alpha_{i} \varepsilon x+z_{i}\right)\right\|_{t, \infty} \\
\geq\left\|\varphi_{t}(\alpha \varepsilon x+z)-\varphi_{\varepsilon, t}^{n}(\alpha, z)\right\|_{t, \infty}-\left(f_{n}(x)+f(x)\right) \cdot l .
\end{gathered}
$$

On en déduit:

$$
\begin{aligned}
& \sup _{i=1, \ldots, m}\left\|\varphi_{t}\left(\alpha_{i} \varepsilon x+z_{i}\right)-\varphi_{\varepsilon, t}^{n}\left(\alpha_{i}, z_{i}\right)\right\|_{t, \infty} \\
& \quad \geq \sup _{(\alpha, z) \in K}\left\|\varphi_{t}(\alpha \varepsilon x+z)-\varphi_{\varepsilon, t}^{n}(\alpha, z)\right\|_{t, \infty}-\left(f_{n}(x)+f(x)\right) \cdot l .
\end{aligned}
$$

Par suite:

$$
\begin{aligned}
\left\{\sup _{(\alpha, z) \in K}\left\|\varphi_{t}(\alpha \varepsilon x+z)-\varphi_{\varepsilon, t}^{n}(\alpha, z)\right\|_{t, \infty} \geq \rho\right\} \\
\subset \bigcup_{i=1}^{m}\left\{\left\|\varphi_{t}\left(\alpha_{i} \varepsilon x+z_{i}\right)-\varphi_{\varepsilon, t}^{n}\left(\alpha_{i}, z_{i}\right)\right\|_{t, \infty} \geq \frac{\rho}{2}\right\} \\
\cup\left\{f_{n} \geq \frac{\rho}{4 l}\right\} \cup\left\{f \geq \frac{\rho}{4 l}\right\} .
\end{aligned}
$$

D'après (4.3) et (4.5):

$$
\begin{array}{r}
\mu\left(\left\{\sup _{(\alpha, z) \in K}\left\|\varphi_{t}(\alpha \varepsilon x+z)-\varphi_{\varepsilon, t}^{n}(\alpha, z)\right\|_{t, \infty} \geq \rho\right\}\right) \\
\leq m e^{-L / \varepsilon^{2}}+C \cdot l / \rho \leq C l^{-d-1} e^{-L / \varepsilon^{2}}+C l / \rho .
\end{array}
$$

En prenant $l=e^{-L / 2(d+1) \varepsilon^{2}}$, on obtient:

$$
\mu\left(\left\{\sup _{(\alpha, z) \in K}\left\|\varphi_{t}(\alpha \varepsilon x+z)-\varphi_{\varepsilon, t}^{n}(\alpha, z)\right\|_{t, \infty} \geq \rho\right\}\right) \leq C \cdot e^{-L / \varepsilon^{2}}
$$

On en déduit donc le Lemme 4.2.

Lemme 4.3. Soit $\rho>0$, on a:

$$
\begin{aligned}
\lim _{n \rightarrow+\infty} \varlimsup_{\varepsilon \rightarrow 0} \varepsilon^{2} \log \mu\left(\left\{\sup _{\alpha \leq 1} \sup _{z \in B_{H_{2}}\left(h_{0}, r\right)}\left\|\varphi_{\varepsilon, t}^{n}(\alpha, z)(y)-\varphi_{t}(\alpha \varepsilon y+z)\right\|_{t, \infty} \geq \rho\right\}\right. \\
\left.\times \varepsilon^{-1} B_{H_{2}}\left(h_{0}, \delta\right)\right)=-\infty .
\end{aligned}
$$


Démonstration. On choisit $\delta, r, R^{\prime \prime}$ tels que $\left\|h_{0}\right\|+\delta+r<R^{\prime \prime}$, alors:

$$
\begin{gathered}
\left\{\sup _{\alpha \leq 1} \sup _{z \in B_{H_{2}}\left(h_{0}, r\right)}\left\|\varphi_{\varepsilon, t}^{n}(\alpha, z)(y)-\varphi_{t}(\alpha \varepsilon y+z)\right\|_{t, \infty} \geq \rho\right\} \times \varepsilon^{-1} B_{H_{2}}\left(h_{0}, \delta\right) \\
\subset\left\{\sup _{(\alpha, z) \in K}\left\|\varphi_{\varepsilon, t}^{n}(\alpha, z)-\varphi_{t}(\alpha \varepsilon x+z)\right\|_{t, \infty} \geq \rho\right\} .
\end{gathered}
$$

Compte tenu du Lemme 4.2, on obtient donc le résultat.

Démonstration du Lemme 3.5. Notons:

$$
\begin{aligned}
G_{\varepsilon}^{n}(y)=\sup _{\alpha \leq 1} \sup _{z \in B_{H_{2}}\left(h_{0}, r\right)}[ & \left\|\varphi_{\varepsilon, t}^{n}(\alpha, z)(y)-\varphi_{t}(\alpha \varepsilon y+z)\right\|_{t, \infty} \\
+ & \left\|Z_{\varepsilon, t}^{n^{-1}}(\alpha, z)(y)-Z_{t}^{-1}(\alpha \varepsilon y+z)\right\|_{t, \infty} \\
+ & \left\|Z_{\varepsilon, t}^{n}(\alpha, z)(y)-Z_{t}(\alpha \varepsilon y+z)\right\|_{t, \infty} \\
& \left.\quad+\left\|\partial_{z} Z_{\varepsilon, t}^{n}(\alpha, z)(y)-\partial_{z} Z_{t}(\alpha \varepsilon y+z)\right\|_{t, \infty}\right] .
\end{aligned}
$$

Alors:

$$
\begin{aligned}
&\left(\left\{I_{\varepsilon} \geq \rho\right\} \times \varepsilon^{-1} B_{H_{2}}\left(h_{0}, \delta\right)\right) \cap\{|\varphi(\varepsilon x)-\xi| \leq \sigma\} \\
& \subset\left(\left\{I_{\varepsilon} \geq \rho, G_{\varepsilon}^{n} \leq \eta\right\} \times \varepsilon^{-1} B_{H_{2}}\left(h_{0}, \delta\right)\right) \cap\{|\varphi(\varepsilon x)-\xi| \leq \sigma\} \\
& \cup\left(\left\{G_{\varepsilon}^{n} \geq \eta\right\} \times \varepsilon^{-1} B_{H_{2}}\left(h_{0}, \delta\right)\right) \\
& \subset\left(\left\{I_{\varepsilon}^{n}\left(\Pi_{Y}(x)\right) \geq \rho-\eta\right\} \cap\left\{\left|\varphi_{1}^{n}(\varepsilon x)-\xi\right| \leq \sigma+\eta\right\}\right. \\
& \cup\left(\left\{G_{\varepsilon}^{n} \geq \eta\right\} \times \varepsilon^{-1} B_{H_{2}}\left(h_{0}, \delta\right)\right) .
\end{aligned}
$$

Où $I_{\varepsilon}^{n}$ est obtenu de $I_{\varepsilon}$ en remplaçant $\varphi_{t}$ par $\varphi_{\varepsilon, t}^{n}(\alpha, z)$ ainsi que leurs dérivées, $\varphi_{1}^{n}(\varepsilon x)=\varphi_{\varepsilon, 1}^{n}(1,0)$. On voit que $x \rightarrow I_{\varepsilon}^{n}\left(\Pi_{Y}(x)\right), x \rightarrow \varphi_{1}^{n}(x)$ sont des fonctions continues sur $X$, en appliquant le principe de grande déviation:

$$
\begin{aligned}
\varlimsup_{\varepsilon \rightarrow 0} 2 \varepsilon^{2} \log \mu\left(\left\{I_{\varepsilon}^{n}\left(\Pi_{Y}(x)\right) \geq \rho-\eta,\left|\varphi_{1}^{n}(\varepsilon x)-\xi\right| \leq \sigma+\eta\right\}\right) \\
=-\inf \left\{\|h\|_{H}^{2}\left|I_{\varepsilon}^{n}\left(P_{H_{1}}(h)\right) \geq \rho-\eta,\right| \varphi_{1}^{n}(h)-\xi \mid \leq \sigma+\eta\right\} .
\end{aligned}
$$

D'àpres le Lemme 4.3 et (4.8), il existe $n_{0}>0$ tel que pour tout $n>n_{0}$ :

$$
\begin{array}{r}
\varlimsup_{\varepsilon \rightarrow 0} 2 \varepsilon^{2} \log \mu\left(\left\{I_{\varepsilon} \geq \rho\right\} \times \varepsilon^{-1} B_{H_{2}}\left(h_{0}, \delta\right) \cap\{|\varphi(\varepsilon x)-\xi| \leq \sigma\}\right) \\
\leq-\inf \left\{\|h\|_{H}^{2}\left|I^{n}\left(P_{H_{1}}(h)\right) \geq \rho-\eta,\right| \varphi_{1}^{n}(h)-\xi \mid \leq \sigma+\eta\right\} .
\end{array}
$$

Posons

$$
W_{n}=\bigcap_{n_{0} \leq m \leq n}\left\{I^{n}\left(P_{H_{1}}(h)\right) \geq \rho-\eta,\left|\varphi_{1}^{n}(h)-\xi\right| \leq \sigma+\eta\right\},
$$

et $c_{n}=\inf \left\{\|h\|_{H}^{2} \mid h \in W_{n}\right\}, c_{0}=\inf \left\{\|h\|_{H}^{2} \mid h \in \bigcap_{n \geq n_{0}} W_{n}\right\}$, alors on a:

$$
c_{0}=\sup _{n \geq n_{0}} c_{n} \text {. }
$$

D'autre part:

$$
\bigcap_{n \geq n_{0}} W_{n} \subset\left\{I_{1}\left(P_{H_{1}}(h)\right) \geq \rho-\eta,|\varphi(h)-\xi| \leq \sigma+\eta\right\}
$$


Il en résulte que:

$$
\begin{gathered}
\varlimsup_{\varepsilon \rightarrow 0} 2 \varepsilon^{2} \log \mu\left(\left(\left\{I_{\varepsilon} \geq \rho\right\} \times \varepsilon^{-1} B_{H_{2}}\left(h_{0}, \delta\right)\right) \cap\{|\varphi(\varepsilon x)-\xi| \leq \sigma\}\right) \\
\leq-\inf \left\{\|h\|_{H}^{2}\left|I_{1}\left(P_{H_{1}}(h)\right) \geq \rho-\eta,\right| \varphi(h)-\xi \mid \leq \sigma+\eta\right\},
\end{gathered}
$$

pour tout $\eta>0$ assez petit. D'où le Lemme 3.5.

\section{BIBLIOGRAPHIE}

[A-M] H. Airault et P. Malliavin, Intégration géométrique sur lespace de Wiener, Bull. Sci. Math (2) 112 (1988), 3-52.

[AZ $\left.\mathrm{A}_{1}\right] \mathrm{R}$. Azencott, Grandes déviations et applications, Ecole d'été de Saint-Flour VIII, Lecture Notes 774, 1980.

$\left[\mathrm{AZ}_{2}\right] \ldots$ Formule de Taylor stochastique et développements asymptotiques de Feynmann, Sém. Probabilité XVI, 1980-1981, Lecture Notes 921, pp. 237-284.

[BE] G. Ben Arous, Développements asmptotiques du noyau de la chaleur hypoelliptique hors du cut-locus, Ann. Ecole Norm. Sup. 21 (1988), 307-331.

[BI $\left.{ }_{1}\right]$ J. M. Bismut, Large deviation and Malliavin calculus, Progr. Math., no. 45, Birkhäuser, Basel, 1984.

[BI $\mathrm{BI}_{2}{ }_{-}$, Mécanique aléatoire, Lecture Notes in Math., vol. 866, Springer-Verlag, Berlin and New York, 1981.

[DO] H. Doss, Démonstration probabiliste de certains développements asymptotiques quasi classiques, Bull. Sci. Math. (2) 109 (1985), 179-208.

[E-R] R. S. Ellis et J. S. Rosen, Asymptotic analysis of Gaussian integrals. I. Isolated minimum points, Trans. Amer. Math. Soc. 273, (1982), 447-481.

[ER] A. Erdelyi, Asymptotics expansions, Dover, New York, 1956.

[FA] S. Fang, Le calcul différentiel quasi-sûr et son application à lestimation du noyau de la chaleur, C. R. Acad. Sci. Paris Sér. I Math. 311 (1990), 369-372.

[HO] L. Hörmander, Hypoelliptic second order differential equations, Acta Math. 119 (1969), 147171.

[I-W] N. Ikeda et S. Watanabe, Stochastic differential equations and diffusions processes, NorthHolland, Amsterdam, 1981.

[K-S] S. Kusuoka et D. W. Stroock, Applications of the Malliavin calculus. II, J. Fac. Sci. Univ. Tokyo Sect. 1A Math. 32 (1985), 1-76.

[LE] R. Leandre, Intégration dans la fibre associée à une diffusion dégénérée, Probab. Theory Related Fields 76 (1987), 341-358.

[MA $\mathbf{M}_{1}$ ] P. Malliavin, Stochastic calculus of variation and hypoeliptic operators, Proc. Int. Sympos. Stochastic Differential Equations, Kyoto, 1976 (K. Ito, ed.), Kinokuniya, 1978, pp. 195-263.

[MA $\left.\mathrm{MA}_{2}\right]$, Implicit functions theorem in finite corank on the Wiener space, Taniguechi Sympos. SA-Katata 1982 (K. Ito, ed.), 1984, pp. 369-386.

[MA3] _ Differential analysis in stochastic analysis, Course at MIT, 1984.

[ $\left.\mathrm{MA}_{4}\right]$ _ Géométrie différentielle stochastique, Presses Univ. Montréal, Montréal, 1978.

[Mc] H. P. McKean, Stochastic integrals, Academic Press, 1969.

[MO] J. M. Moulinier, Théorème limite pour les équations différentielles stochastiques, Bull. Sci. Math. (2) 112 (1988), 185-208.

[O-P] D. Ocone et E. Pardoux, A generalized Itô-Ventzell formula. Application to a class of anticipating stochastic differential equations, Ann. Inst. H. Poincaré Probab. Statist. 25 (1989), 39-71.

[RE] J. Ren, Analyse stochatique quasi-sûre des équations différentielles stochastiques, Bull. Sci. Math. (2) 114, 90, 187-213.

[ST] D. W. Stroock, An introduction to the théory of large deviation, Springer, New York, 1988. 
[S-V] D. W. Stroock et S. R. S. Varadhan, On the support on diffusion processes with applications to the strong maximum principle, Proc. 6th Berkeley Sympos. on Math. Stat. and Prob., vol. 3, Univ. of California Press, Berkeley, 1972, pp. 335-359.

[SU] H. Sugita, Positive generalized Wiener functions and potential theory over abstract Wiener spaces, Osaka J. Math. 25 (1988), 665-696.

[WA $\mathrm{W}_{1}$ S. Watanabe, Lectures on stochastic differential equations and Malliavin calculus, Tata Institute of Fundamental Research, Bombay, 1984.

[WA $\mathrm{W}_{2}$ ] _ Analysis of Wiener functionals (Malliavin calculus) and applications to heat kernels, Ann. Probab. 15 (1987), 1-39.

Géométrie et Analyse Complexe, $5^{e}$ étage, 46-0, Université de Paris Vi, 75253 Paris, Cedex 05, France

Department of Mathematics, University of Wuhan, Wuhan, China 